\title{
Y-aromaticity: Why is the Trimethylene Methane Dication More Stable than the Butadienyl Dication?
}

\author{
Amy Dworkin, Rachel Naumann, Christopher Seigfred and Joel M. Karty* \\ Department of Chemistry, Elon University \\ 2625 Campus Box \\ Elon, NC 27244 \\ jkarty@elon.edu
}

\section{Contents}

Pages S1-S51 contain the Cartesian coordinates and the thermally corrected enthalpies (B3LYP/6-31+G*, 298K) of all species involved in the vinylogue methodology. Pages S52-S57 contain the Cartesian coordinates and energies of the species in the orbital deletion procedure (ODP) methodology.

Note: All perpendicular vinylogues have 1 imaginary frequency. All others have 0 imaginary frequencies. 


\section{Y-shape Neutral}

\begin{tabular}{|c|c|c|c|c|c|}
\hline & Enthalpy & \multicolumn{2}{|c|}{$-157.119728 \mathrm{H}$} & & \multirow{2}{*}{----------} \\
\hline \multicolumn{5}{|l|}{--} & \\
\hline Center & Atomic & Atomic & \multicolumn{3}{|c|}{ Coordinates (Angstroms) } \\
\hline Number & Number & Type & $\mathrm{X}$ & $\mathrm{Y}$ & $\mathrm{Z}$ \\
\hline \multicolumn{6}{|c|}{ 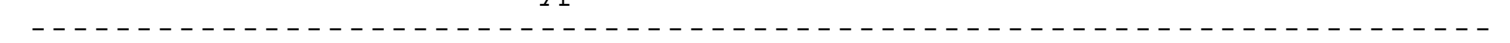 } \\
\hline 1 & 6 & 0 & 0.000000 & 0.122965 & -0.000045 \\
\hline 2 & 6 & 0 & 0.000000 & 1.462980 & 0.000000 \\
\hline 3 & 1 & 0 & 0.925574 & 2.034542 & 0.000035 \\
\hline 4 & 1 & 0 & -0.925573 & 2.034543 & 0.000036 \\
\hline 5 & 6 & 0 & 1.278349 & -0.680543 & 0.000002 \\
\hline 6 & 1 & 0 & 1.330581 & -1.335514 & 0.881270 \\
\hline 7 & 1 & 0 & 2.164525 & -0.037603 & -0.000279 \\
\hline 8 & 1 & 0 & 1.330350 & -1.336005 & -0.880904 \\
\hline 9 & 6 & 0 & -1.278349 & -0.680543 & 0.000002 \\
\hline 10 & 1 & 0 & -1.330580 & -1.335515 & 0.881269 \\
\hline 11 & 1 & 0 & -1.330351 & -1.336003 & -0.880905 \\
\hline 12 & 1 & 0 & -2.164525 & -0.037602 & -0.000278 \\
\hline$\ldots$ & & & --------- & -------- & --------- \\
\hline
\end{tabular}

\section{Y-shape Dication}

\begin{tabular}{|c|c|c|c|c|c|}
\hline $\mathrm{n}=0$ & \multicolumn{3}{|c|}{ Enthalpy $=-155.033034 \mathrm{H}$} & & \\
\hline \multicolumn{6}{|c|}{ - - - - - - - - - - - - - - - - - - - - - - - - - - - - - - - - - - - - - - - - - - - - - - - - - - - - } \\
\hline Center & Atomic & Atomic & Coord & lates & roms ) \\
\hline Number & Number & Type & $\mathrm{X}$ & $\mathrm{Y}$ & Z \\
\hline \multicolumn{6}{|c|}{ 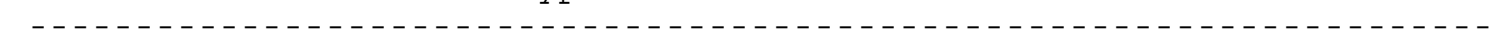 } \\
\hline 1 & 6 & 0 & -0.000050 & -0.000040 & -0.000011 \\
\hline 2 & 6 & 0 & -1.377510 & -0.366399 & 0.000024 \\
\hline 3 & 1 & 0 & -1.693838 & -1.414710 & -0.000405 \\
\hline 4 & 1 & 0 & -2.172774 & 0.386309 & 0.000169 \\
\hline 5 & 6 & 0 & 1.006087 & -1.009656 & -0.000037 \\
\hline 6 & 1 & 0 & 2.072043 & -0.759160 & 0.000219 \\
\hline 7 & 1 & 0 & 0.752207 & -2.074823 & 0.000292 \\
\hline 8 & 6 & 0 & 0.371420 & 1.376059 & -0.000027 \\
\hline 9 & 1 & 0 & 1.420940 & 1.688403 & -0.000278 \\
\hline 10 & 1 & 0 & -0.378261 & 2.174189 & 0.000301 \\
\hline
\end{tabular}




\section{Y-shape Parallel Neutral}

\begin{tabular}{|c|c|c|c|c|c|c|}
\hline \multicolumn{7}{|c|}{$\begin{array}{l}\mathrm{n}=1 \quad \text { Enthalpy }=-311.850551 \mathrm{H} \\
-\ldots\end{array}$} \\
\hline \multirow{2}{*}{$\begin{array}{l}\text { Center } \\
\text { Number }\end{array}$} & \multirow{2}{*}{$\begin{array}{l}\text { Atomi } \\
\text { Number }\end{array}$} & \multirow{2}{*}{$\begin{array}{l}\text { Atomic } \\
\text { Type }\end{array}$} & \multirow{3}{*}{\multicolumn{2}{|c|}{$\begin{array}{l}\text { Coordinates } \\
\mathrm{X}\end{array}$}} & \multirow{2}{*}{$\begin{array}{l}\text { (Angstroms) } \\
\mathrm{Y}\end{array}$} & \multirow[b]{2}{*}{$\mathrm{Z}$} \\
\hline & & & & & & \\
\hline------- & 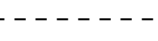 & --- & & & ------------ & ---------- \\
\hline 1 & 6 & & 0 & 0.000000 & 0.478722 & 0.000000 \\
\hline 2 & 6 & & 0 & 0.000000 & 1.829492 & 0.000000 \\
\hline 3 & 1 & & 0 & -0.920098 & 2.405235 & 0.000094 \\
\hline 4 & 1 & & 0 & 0.920098 & 2.405235 & -0.000093 \\
\hline 5 & 6 & & 0 & 1.245243 & -0.328786 & 0.000006 \\
\hline 6 & 1 & & 0 & 1.088925 & -1.408871 & 0.000022 \\
\hline 7 & 6 & & 0 & 2.515633 & 0.106625 & 0.000010 \\
\hline 8 & 1 & & 0 & 2.721056 & 1.177796 & 0.000053 \\
\hline 9 & 6 & & 0 & -1.245243 & -0.328786 & -0.000007 \\
\hline 10 & 1 & & 0 & -1.088925 & -1.408871 & -0.000023 \\
\hline 11 & 6 & & 0 & -2.515633 & 0.106625 & -0.000010 \\
\hline 12 & 1 & & 0 & -2.721056 & 1.177796 & -0.000053 \\
\hline 13 & 6 & & 0 & 3.722366 & -0.786459 & 0.000000 \\
\hline 14 & 1 & & 0 & 3.441640 & -1.845705 & -0.000199 \\
\hline 15 & 1 & & 0 & 4.353131 & -0.600811 & 0.880853 \\
\hline 16 & 1 & & 0 & 4.353236 & -0.600565 & -0.880735 \\
\hline 17 & 6 & & 0 & -3.722366 & -0.786459 & 0.000001 \\
\hline 18 & 1 & & 0 & -3.441640 & -1.845705 & 0.000198 \\
\hline 19 & 1 & & 0 & -4.353132 & -0.600811 & -0.880852 \\
\hline 20 & 1 & & 0 & -4.353235 & -0.600566 & 0.880737 \\
\hline
\end{tabular}




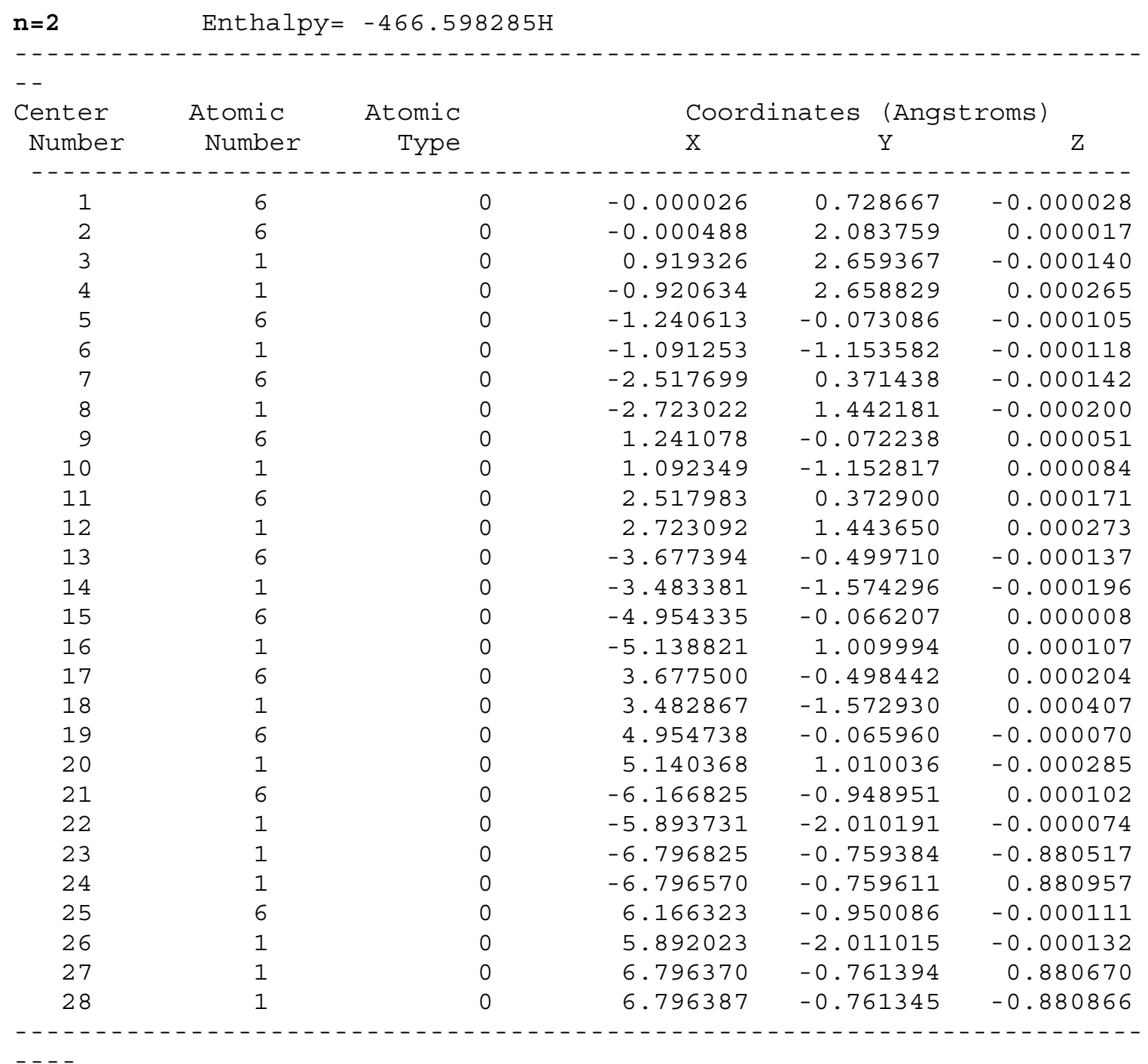




\begin{tabular}{|c|c|c|c|c|c|}
\hline $\mathrm{n}=3$ & \multicolumn{2}{|c|}{ Enthalpy $=-621.346344$} & -------- & -------1 & ----- \\
\hline \multirow{2}{*}{$\begin{array}{l}\text { Center } \\
\text { Number }\end{array}$} & \multirow{3}{*}{$\begin{array}{l}\text { Atomic } \\
\text { Number }\end{array}$} & \multirow{3}{*}{$\begin{array}{l}\text { Atomic } \\
\text { Type }\end{array}$} & \multicolumn{3}{|c|}{ Coordinates (Angstroms) } \\
\hline & & & $\mathrm{X}$ & $\mathrm{Y}$ & $\mathrm{Z}$ \\
\hline------ & & & -------- & -------1 & -------- \\
\hline 1 & 6 & 0 & 0.000005 & 0.936209 & -0.000156 \\
\hline 2 & 6 & 0 & -0.000051 & 2.291816 & -0.000169 \\
\hline 3 & 1 & 0 & 0.893311 & 2.861166 & 0.237824 \\
\hline 4 & 1 & 0 & -0.893472 & 2.861044 & -0.238224 \\
\hline 5 & 6 & 0 & -1.229135 & 0.148761 & -0.206876 \\
\hline 6 & 1 & 0 & -1.087835 & -0.854576 & -0.612132 \\
\hline 7 & 6 & 0 & -2.492100 & 0.545232 & 0.082454 \\
\hline 8 & 1 & 0 & -2.650093 & 1.519984 & 0.545549 \\
\hline 9 & 6 & 0 & 1.229140 & 0.148757 & 0.206586 \\
\hline 10 & 1 & 0 & 1.087772 & -0.854816 & 0.611229 \\
\hline 11 & 6 & 0 & 2.492191 & 0.545547 & -0.081924 \\
\hline 12 & 1 & 0 & 2.650288 & 1.520573 & -0.544408 \\
\hline 13 & 6 & 0 & -3.670532 & -0.251624 & -0.165894 \\
\hline 14 & 1 & 0 & -3.518274 & -1.229207 & -0.626875 \\
\hline 15 & 6 & 0 & -4.935315 & 0.141219 & 0.133944 \\
\hline 16 & 1 & 0 & -5.085860 & 1.119959 & 0.593264 \\
\hline 17 & 6 & 0 & 3.670607 & -0.251384 & 0.166148 \\
\hline 18 & 1 & 0 & 3.518387 & -1.229047 & 0.626978 \\
\hline 19 & 6 & 0 & 4.935362 & 0.141336 & -0.133968 \\
\hline 20 & 1 & 0 & 5.085920 & 1.120108 & -0.593195 \\
\hline 21 & 6 & 0 & -6.121528 & -0.651406 & -0.115198 \\
\hline 22 & 1 & 0 & -5.968952 & -1.629704 & -0.575777 \\
\hline 23 & 6 & 0 & -7.377689 & -0.259810 & 0.183138 \\
\hline 24 & 1 & 0 & -7.522075 & 0.719838 & 0.643294 \\
\hline 25 & 6 & 0 & 6.121494 & -0.651453 & 0.115063 \\
\hline 26 & 1 & 0 & 5.968829 & -1.629819 & 0.575404 \\
\hline 27 & 6 & 0 & 7.377686 & -0.259768 & -0.183091 \\
\hline 28 & 1 & 0 & 7.522226 & 0.719977 & -0.642996 \\
\hline 29 & 6 & 0 & -8.616547 & -1.066997 & -0.066304 \\
\hline 30 & 1 & 0 & -8.383270 & -2.032016 & -0.530105 \\
\hline 31 & 1 & 0 & -9.314025 & -0.531736 & -0.726217 \\
\hline 32 & 1 & 0 & -9.160017 & -1.261291 & 0.869325 \\
\hline 33 & 6 & 0 & 8.616458 & -1.067190 & 0.066275 \\
\hline 34 & 1 & 0 & 8.382957 & -2.032266 & 0.529821 \\
\hline 35 & 1 & 0 & 9.313944 & -0.532237 & 0.726433 \\
\hline 36 & 1 & 0 & 9.159963 & -1.261411 & -0.869351 \\
\hline
\end{tabular}




\begin{tabular}{|c|c|c|c|c|c|}
\hline \multicolumn{6}{|c|}{$\begin{array}{l}\mathrm{n}=4 \quad \text { Enthalpy }=-776.095720 \mathrm{H} \\
--\end{array}$} \\
\hline Center & Atomic & \multirow{2}{*}{$\begin{array}{l}\text { Atomic } \\
\text { Type }\end{array}$} & \multicolumn{3}{|c|}{ Coordinates (Angstroms) } \\
\hline Number & Number & & $\mathrm{X}$ & Y & $\mathrm{Z}$ \\
\hline \multicolumn{6}{|c|}{ - - - - - - - - - - - - - - - - - - - - - - - - - - - - - - - - - - - - - - - - - - - - - - - - - - - - - - - - } \\
\hline 1 & 6 & 0 & -0.000044 & 1.154429 & 0.000055 \\
\hline 2 & 6 & 0 & -0.000342 & 2.512059 & -0.000275 \\
\hline 3 & 1 & 0 & -0.920228 & 3.087197 & -0.000403 \\
\hline 4 & 1 & 0 & 0.919342 & 3.087521 & -0.000411 \\
\hline 5 & 6 & 0 & -1.238760 & 0.355593 & 0.000240 \\
\hline 6 & 1 & 0 & -1.093066 & -0.725181 & 0.000406 \\
\hline 7 & 6 & 0 & -2.518715 & 0.805513 & 0.000150 \\
\hline 8 & 1 & 0 & -2.717091 & 1.877482 & -0.000044 \\
\hline 9 & 6 & 0 & -3.673680 & -0.053709 & 0.000236 \\
\hline 10 & 1 & 0 & -3.491871 & -1.129854 & 0.000314 \\
\hline 11 & 6 & 0 & -4.960572 & 0.393345 & 0.000167 \\
\hline 12 & 1 & 0 & -5.139152 & 1.470199 & 0.000045 \\
\hline 13 & 6 & 0 & -6.120309 & -0.458538 & 0.000189 \\
\hline 14 & 1 & 0 & -5.942173 & -1.535478 & 0.000279 \\
\hline 15 & 6 & 0 & -7.404174 & -0.009715 & 0.000082 \\
\hline 16 & 1 & 0 & -7.583227 & 1.067047 & -0.000001 \\
\hline 17 & 6 & 0 & -8.570665 & -0.865348 & 0.000041 \\
\hline 18 & 1 & 0 & -8.388334 & -1.942011 & 0.000239 \\
\hline 19 & 6 & 0 & -9.845111 & -0.420250 & -0.000226 \\
\hline 20 & 1 & 0 & -10.020019 & 0.657515 & -0.000376 \\
\hline 21 & 6 & 0 & -11.064462 & -1.292858 & -0.000447 \\
\hline 22 & 1 & 0 & -11.692227 & -1.099010 & -0.881857 \\
\hline 23 & 1 & 0 & -11.693789 & -1.097320 & 0.879456 \\
\hline 24 & 1 & 0 & -10.799987 & -2.356220 & 0.000772 \\
\hline 25 & 6 & 0 & 1.239087 & 0.356266 & 0.000220 \\
\hline 26 & 1 & 0 & 1.093819 & -0.724571 & 0.000409 \\
\hline 27 & 6 & 0 & 2.518881 & 0.806687 & 0.000111 \\
\hline 28 & 1 & 0 & 2.717162 & 1.878635 & -0.000115 \\
\hline 29 & 6 & 0 & 3.673623 & -0.052898 & 0.000215 \\
\hline 30 & 1 & 0 & 3.491044 & -1.128955 & 0.000332 \\
\hline 31 & 6 & 0 & 4.960883 & 0.392843 & 0.000101 \\
\hline 32 & 1 & 0 & 5.140569 & 1.469528 & -0.000081 \\
\hline 33 & 6 & 0 & 6.119894 & -0.460028 & 0.000127 \\
\hline 34 & 1 & 0 & 5.941284 & -1.536870 & 0.000274 \\
\hline 35 & 6 & 0 & 7.403768 & -0.011243 & -0.000038 \\
\hline 36 & 1 & 0 & 7.582554 & 1.065564 & -0.000169 \\
\hline 37 & 6 & 0 & 8.570670 & -0.866333 & -0.000055 \\
\hline 38 & 1 & 0 & 8.389079 & -1.943114 & 0.000242 \\
\hline 39 & 6 & 0 & 9.844744 & -0.420169 & -0.000497 \\
\hline 40 & 1 & 0 & 10.018474 & 0.657801 & -0.000920 \\
\hline 41 & 6 & 0 & 11.065100 & -1.291241 & -0.000149 \\
\hline 42 & 1 & 0 & 11.695111 & -1.093648 & -0.879081 \\
\hline 43 & 1 & 0 & 10.802176 & -2.355018 & -0.002987 \\
\hline 44 & 1 & 0 & 11.691662 & -1.097669 & 0.882194 \\
\hline
\end{tabular}




\section{Y-shape Parallel Dication}

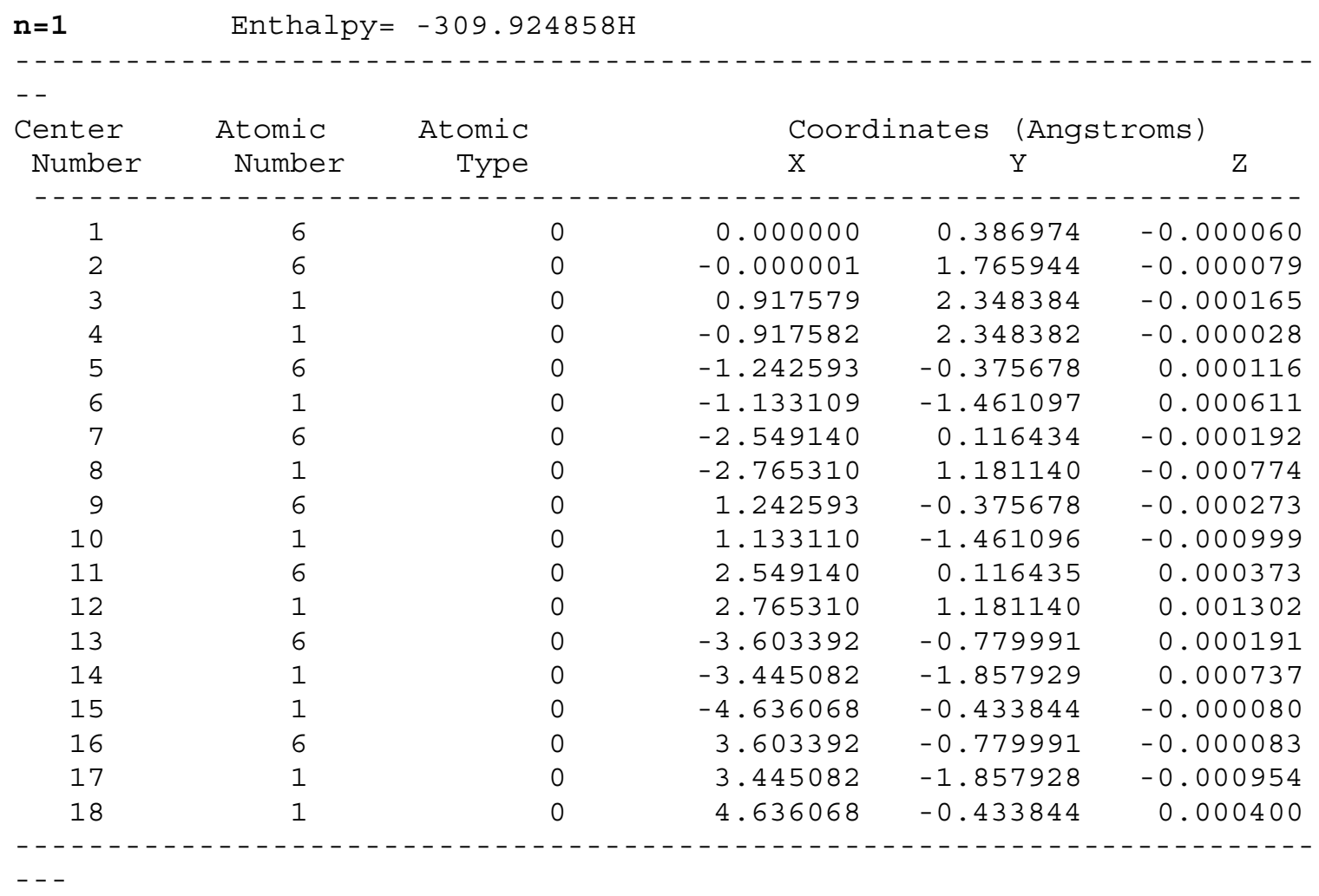




\begin{tabular}{|c|c|c|c|c|c|}
\hline $\mathrm{n}=2$ & Enthalpy= & $-464.757410 \mathrm{H}$ & & & \multirow{2}{*}{-----------} \\
\hline--- & ------ & --1 & & & \\
\hline \multirow{2}{*}{$\begin{array}{l}\text { Center } \\
\text { Number }\end{array}$} & \multirow{2}{*}{$\begin{array}{l}\text { Atomic } \\
\text { Number }\end{array}$} & \multirow{2}{*}{$\begin{array}{r}\text { Atomic } \\
\text { Type }\end{array}$} & \multicolumn{3}{|c|}{ Coordinates (Angstroms) } \\
\hline & & & $\mathrm{X}$ & $\mathrm{Y}$ & Z \\
\hline \multicolumn{6}{|c|}{ - - - - - - - - - - - - - - - - - - - - - - - - - - - - - - - - - - - - - - - - - - - - - - - - - - - - - - - - - - - } \\
\hline 1 & 6 & 0 & -0.000003 & 0.685192 & -0.063060 \\
\hline 2 & 6 & 0 & 0.000023 & 2.052959 & -0.069616 \\
\hline 3 & 1 & 0 & -0.916863 & 2.633434 & -0.083099 \\
\hline 4 & 1 & 0 & 0.916884 & 2.633514 & -0.081508 \\
\hline 5 & 6 & 0 & 1.244424 & -0.086068 & -0.077035 \\
\hline 6 & 1 & 0 & 1.126746 & -1.165194 & -0.176740 \\
\hline 7 & 6 & 0 & 2.530717 & 0.391611 & 0.027852 \\
\hline 8 & 1 & 0 & 2.736156 & 1.452294 & 0.148441 \\
\hline 9 & 6 & 0 & -1.244556 & -0.085840 & -0.078770 \\
\hline 10 & 1 & 0 & -1.127111 & -1.164626 & -0.182384 \\
\hline 11 & 6 & 0 & -2.530651 & 0.391670 & 0.029336 \\
\hline 12 & 1 & 0 & -2.735728 & 1.451862 & 0.154526 \\
\hline 13 & 6 & 0 & 3.628021 & -0.495585 & -0.011405 \\
\hline 14 & 1 & 0 & 3.424993 & -1.561017 & -0.126072 \\
\hline 15 & 6 & 0 & 4.976262 & -0.089361 & 0.087956 \\
\hline 16 & 1 & 0 & 5.209187 & 0.966889 & 0.203705 \\
\hline 17 & 6 & 0 & -3.628085 & -0.495243 & -0.012450 \\
\hline 18 & 1 & 0 & -3.425189 & -1.560215 & -0.131497 \\
\hline 19 & 6 & 0 & -4.976258 & -0.089494 & 0.089681 \\
\hline 20 & 1 & 0 & -5.209175 & 0.966272 & 0.209747 \\
\hline 21 & 6 & 0 & 5.986884 & -1.012618 & 0.038865 \\
\hline 22 & 1 & 0 & 5.786699 & -2.076220 & -0.076622 \\
\hline 23 & 1 & 0 & 7.029614 & -0.713945 & 0.113572 \\
\hline 24 & 6 & 0 & -5.986802 & -1.012687 & 0.037853 \\
\hline 25 & 1 & 0 & -5.786576 & -2.075808 & -0.081929 \\
\hline 26 & 1 & 0 & -7.029503 & -0.714461 & 0.114606 \\
\hline
\end{tabular}




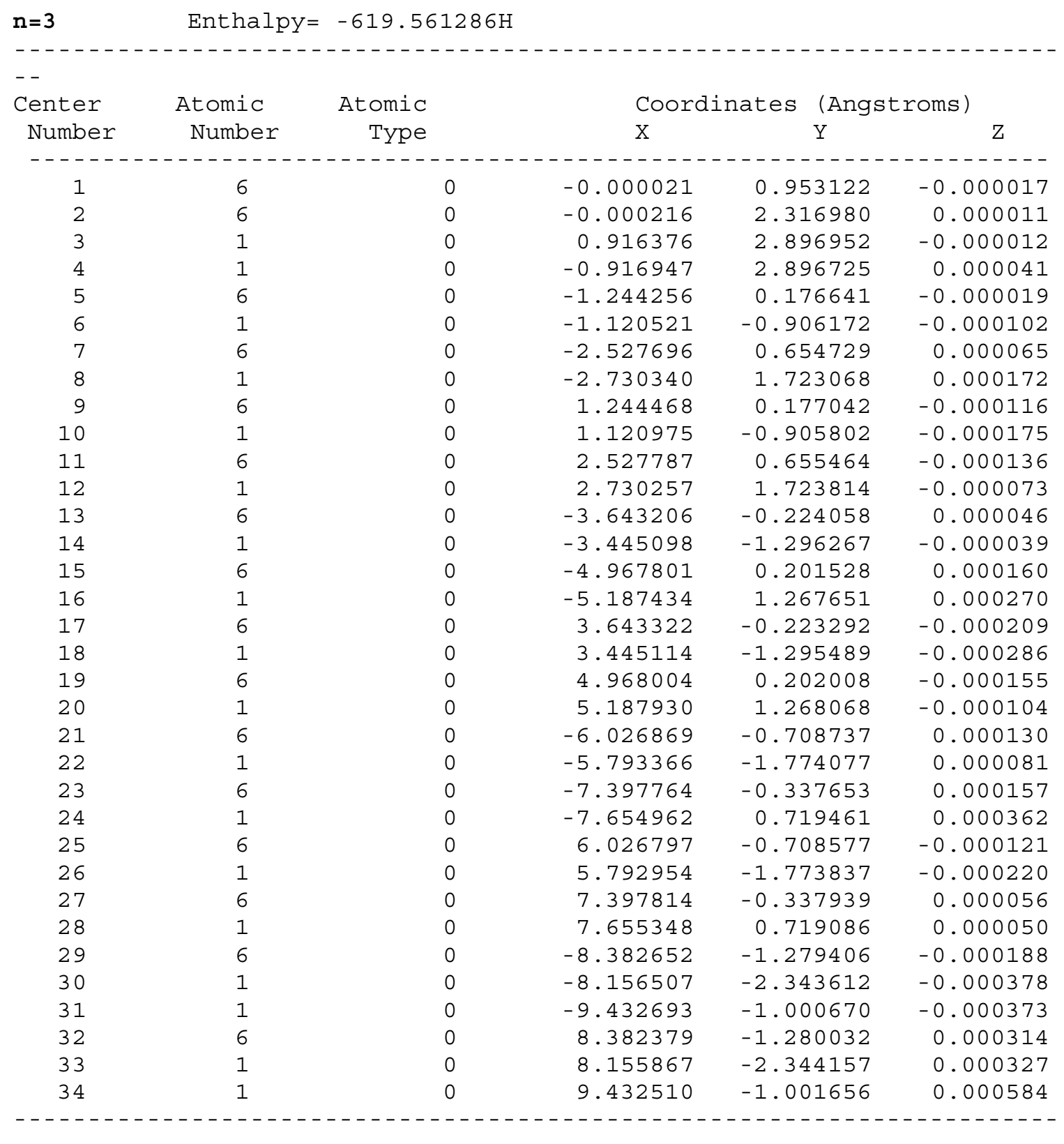




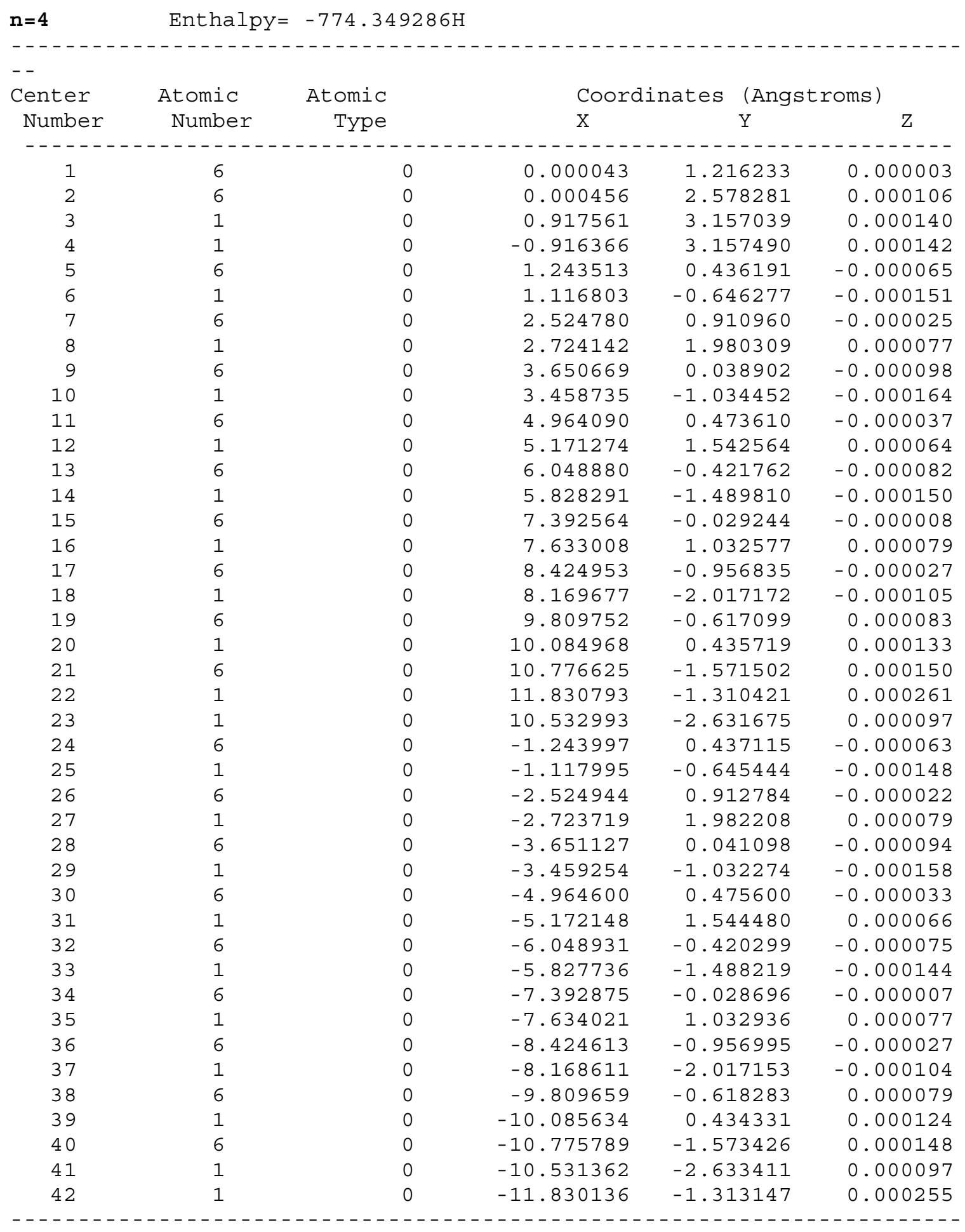




\section{Y-shape Perpendicular Neutral}

\begin{tabular}{|c|c|c|c|c|c|}
\hline \multicolumn{6}{|c|}{$\begin{array}{l}\mathrm{n}=1 \quad \text { Enthalpy }=-311.843207 \mathrm{H} \\
--\end{array}$} \\
\hline Center & Atomic & Atomic & \multicolumn{3}{|c|}{ Coordinates (Angstroms) } \\
\hline Number & Number & Type & $\mathrm{X}$ & $\mathrm{Y}$ & Z \\
\hline \multicolumn{6}{|c|}{-------------------------------------------------------------------} \\
\hline 1 & 6 & 0 & 0.000000 & 0.916138 & 0.000010 \\
\hline 2 & 6 & 0 & -0.000016 & 2.256639 & -0.000019 \\
\hline 3 & 1 & 0 & 0.872749 & 2.822655 & 0.317570 \\
\hline 4 & 1 & 0 & -0.872795 & 2.822620 & -0.317631 \\
\hline 5 & 6 & 0 & -1.185451 & 0.117848 & -0.439060 \\
\hline 6 & 1 & 0 & -1.165092 & -0.229733 & -1.475740 \\
\hline 7 & 6 & 0 & -2.175616 & -0.292345 & 0.363975 \\
\hline 8 & 1 & 0 & -2.168313 & 0.026048 & 1.408267 \\
\hline 9 & 6 & 0 & 1.185480 & 0.117894 & 0.439086 \\
\hline 10 & 1 & 0 & 1.165174 & -0.229625 & 1.475787 \\
\hline 11 & 6 & 0 & 2.175631 & -0.292307 & -0.363962 \\
\hline 12 & 1 & 0 & 2.168300 & 0.026059 & -1.408262 \\
\hline 13 & 6 & 0 & -3.330476 & -1.155941 & -0.061163 \\
\hline 14 & 1 & 0 & -3.263761 & -1.422973 & -1.121946 \\
\hline 15 & 1 & 0 & -4.289562 & -0.645631 & 0.102596 \\
\hline 16 & 1 & 0 & -3.364555 & -2.086235 & 0.522682 \\
\hline 17 & 6 & 0 & 3.330462 & -1.155957 & 0.061148 \\
\hline 18 & 1 & 0 & 4.289622 & -0.646108 & -0.103576 \\
\hline 19 & 1 & 0 & 3.363867 & -2.086714 & -0.522008 \\
\hline 20 & 1 & 0 & 3.264278 & -1.422178 & 1.122169 \\
\hline
\end{tabular}




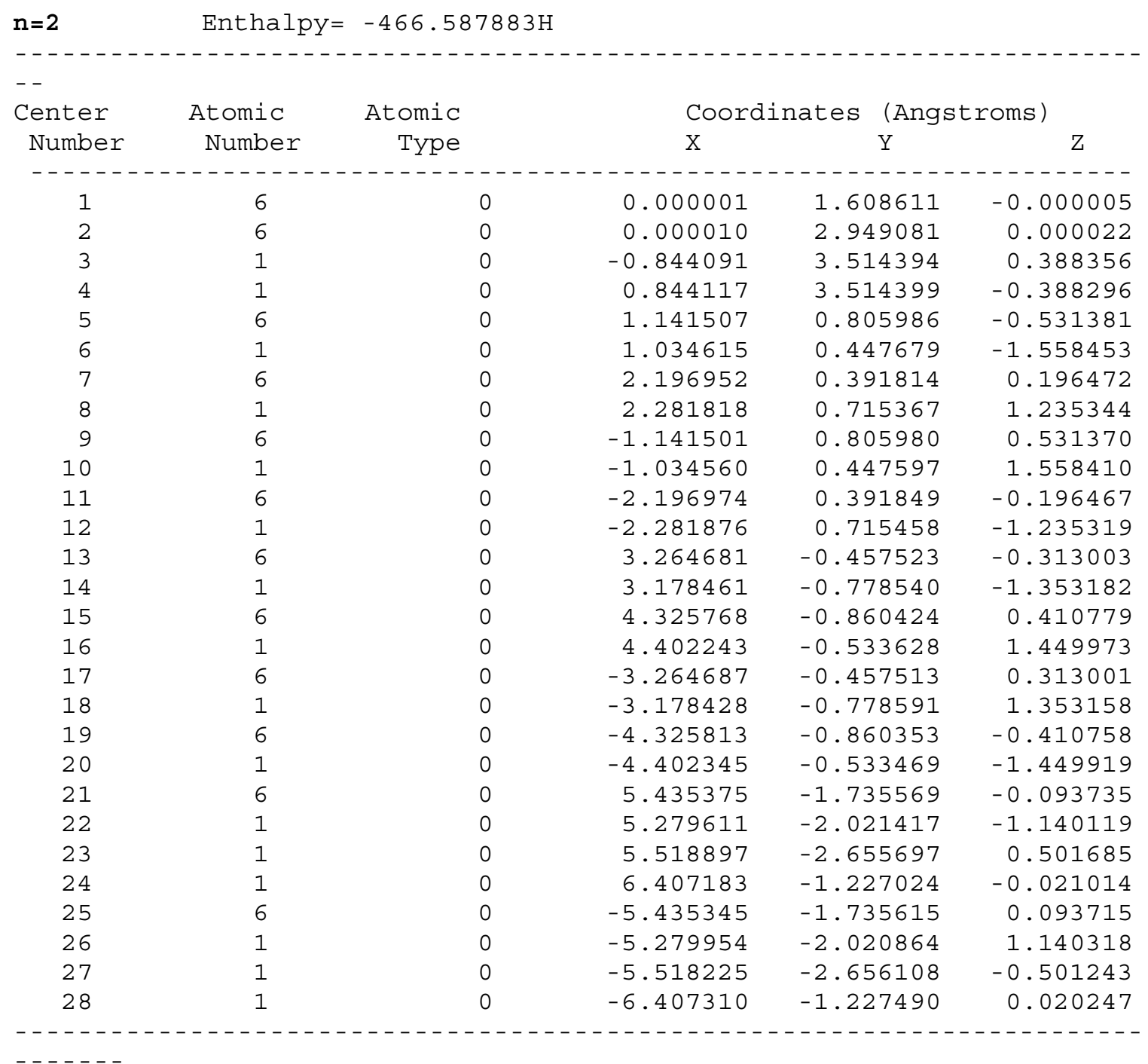




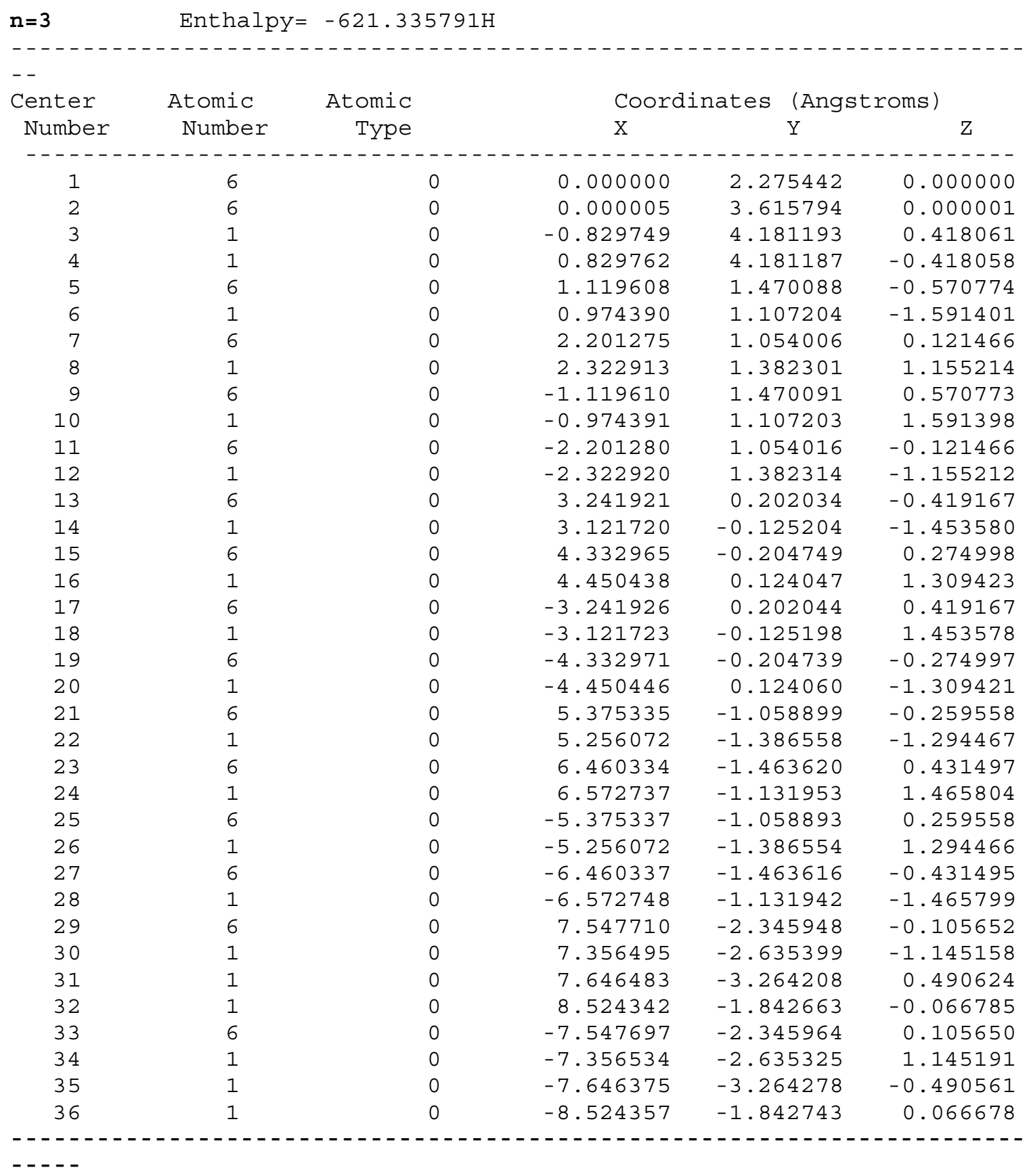




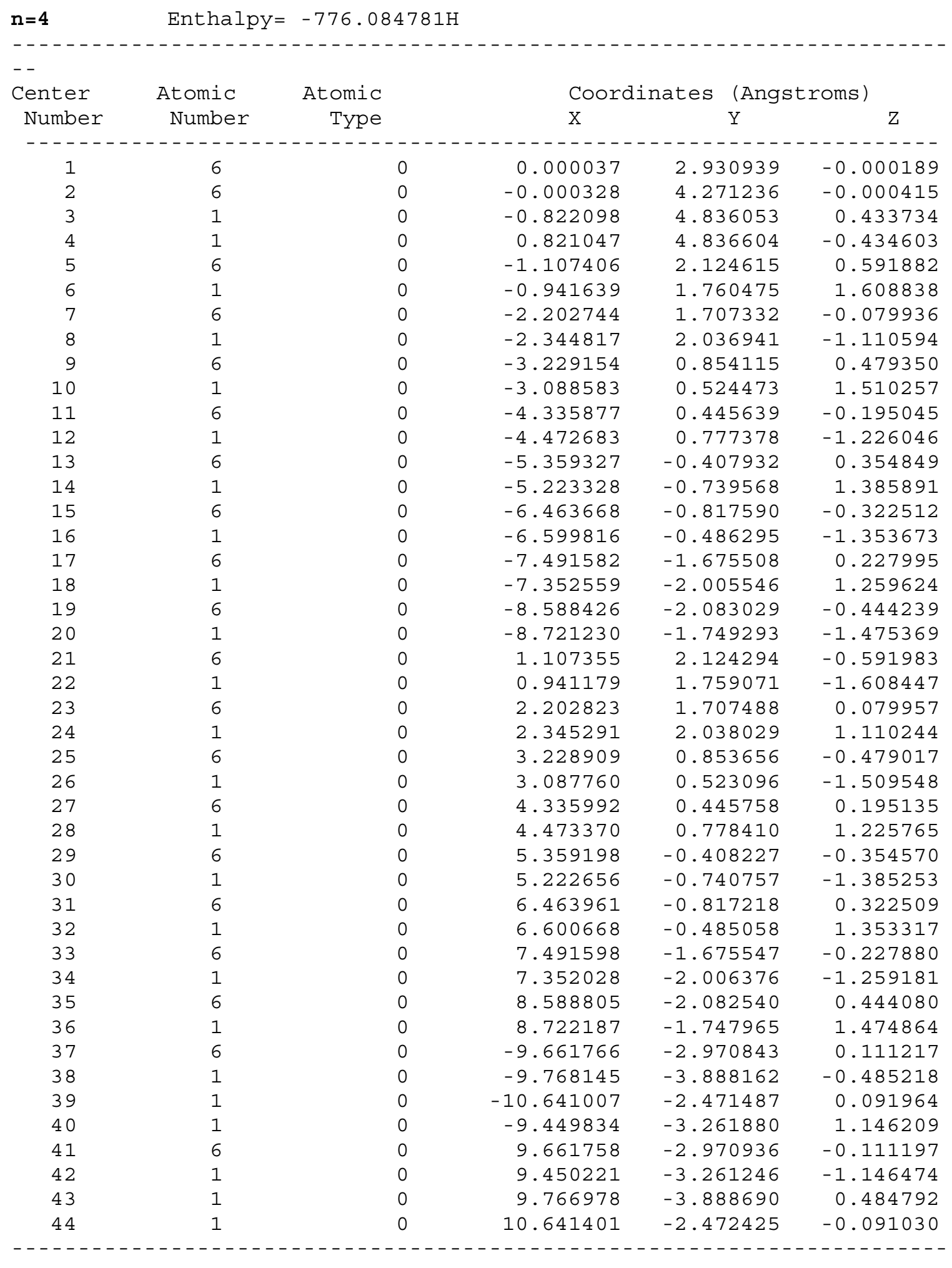




\section{$\underline{\text { Y-shape Perpendicular Dication }}$}

\begin{tabular}{|c|c|c|c|c|c|}
\hline \multirow{2}{*}{\multicolumn{6}{|c|}{$\begin{array}{l}\mathrm{n}=1 \\
--- \\
--\end{array}$}} \\
\hline & & & & & \\
\hline \multirow{2}{*}{$\begin{array}{l}\text { Center } \\
\text { Number }\end{array}$} & \multirow{2}{*}{$\begin{array}{l}\text { Atomic } \\
\text { Number }\end{array}$} & \multirow{2}{*}{$\begin{array}{r}\text { Atomic } \\
\text { Type }\end{array}$} & \multicolumn{3}{|c|}{ Coordinates (Angstroms) } \\
\hline & & & $\mathrm{X}$ & $\mathrm{Y}$ & \multirow{2}{*}{ 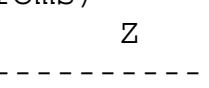 } \\
\hline.---- & ------- & ------- & --------- & --------1 & \\
\hline 1 & 6 & 0 & 0.022560 & 0.808260 & 0.136426 \\
\hline 2 & 6 & 0 & -0.061757 & 2.096804 & -0.278822 \\
\hline 3 & 1 & 0 & 0.769466 & 2.584995 & -0.784221 \\
\hline 4 & 1 & 0 & -0.966003 & 2.686785 & -0.141185 \\
\hline 5 & 6 & 0 & 1.135543 & -0.055657 & -0.306481 \\
\hline 6 & 1 & 0 & 0.931420 & -0.760080 & -1.118037 \\
\hline 7 & 6 & 0 & 2.343566 & -0.173279 & 0.375530 \\
\hline 8 & 1 & 0 & 2.547425 & 0.448524 & 1.244777 \\
\hline 9 & 6 & 0 & 3.285350 & -1.097590 & -0.058198 \\
\hline 10 & 1 & 0 & 4.220120 & -1.244004 & 0.482798 \\
\hline 11 & 1 & 0 & 3.123842 & -1.714959 & -0.941950 \\
\hline 12 & 6 & 0 & -1.247933 & 0.127583 & 0.494804 \\
\hline 13 & 1 & 0 & -1.638490 & 0.375615 & 1.486812 \\
\hline 14 & 6 & 0 & -2.072060 & -0.601329 & -0.358625 \\
\hline 15 & 1 & 0 & -1.777717 & -0.818051 & -1.383353 \\
\hline 16 & 6 & 0 & -3.325523 & -0.985824 & 0.095670 \\
\hline 17 & 1 & 0 & -4.040774 & -1.471259 & -0.568021 \\
\hline 18 & 1 & 0 & -3.647762 & -0.801364 & 1.120556 \\
\hline
\end{tabular}




\begin{tabular}{|c|c|c|c|c|c|}
\hline \multicolumn{6}{|c|}{$\begin{array}{l}-------------------------------------------------------------------- \\
--\end{array}$} \\
\hline Center & Atomic & Atomic & \multicolumn{3}{|c|}{ Coordinates (Angstroms) } \\
\hline Number & Number & Type & $\mathrm{X}$ & $\mathrm{Y}$ & $\mathrm{Z}$ \\
\hline \multicolumn{6}{|c|}{--------------------------------------------------------------------} \\
\hline 1 & 6 & 0 & 0.000008 & 1.423028 & 0.000027 \\
\hline 2 & 6 & 0 & -0.000028 & 2.762560 & -0.000175 \\
\hline 3 & 1 & 0 & 0.855386 & 3.331959 & -0.354378 \\
\hline 4 & 1 & 0 & -0.855468 & 3.332025 & 0.353857 \\
\hline 5 & 6 & 0 & -1.168637 & 0.635857 & 0.493128 \\
\hline 6 & 1 & 0 & -1.167652 & 0.360537 & 1.550783 \\
\hline 7 & 6 & 0 & -2.212321 & 0.228629 & -0.293431 \\
\hline 8 & 1 & 0 & -2.234661 & 0.480041 & -1.352146 \\
\hline 9 & 6 & 0 & 1.168579 & 0.635752 & -0.493065 \\
\hline 10 & 1 & 0 & 1.167335 & 0.359972 & -1.550599 \\
\hline 11 & 6 & 0 & 2.212426 & 0.228819 & 0.293432 \\
\hline 12 & 1 & 0 & 2.234979 & 0.480645 & 1.352044 \\
\hline 13 & 6 & 0 & -3.313532 & -0.468102 & 0.272272 \\
\hline 14 & 1 & 0 & -3.277327 & -0.701521 & 1.337403 \\
\hline 15 & 6 & 0 & -4.455621 & -0.861144 & -0.451826 \\
\hline 16 & 1 & 0 & -4.520406 & -0.640702 & -1.515064 \\
\hline 17 & 6 & 0 & 3.313502 & -0.468173 & -0.272212 \\
\hline 18 & 1 & 0 & 3.277099 & -0.701981 & -1.337251 \\
\hline 19 & 6 & 0 & 4.455709 & -0.860981 & 0.451827 \\
\hline 20 & 1 & 0 & 4.520683 & -0.640164 & 1.514975 \\
\hline 21 & 6 & 0 & -5.486588 & -1.507998 & 0.181632 \\
\hline 22 & 1 & 0 & -5.451602 & -1.738159 & 1.245089 \\
\hline 23 & 1 & 0 & -6.380269 & -1.812440 & -0.358402 \\
\hline 24 & 6 & 0 & 5.486557 & -1.508074 & -0.181582 \\
\hline 25 & 1 & 0 & 5.451303 & -1.738777 & -1.244913 \\
\hline 26 & 1 & 0 & 6.380268 & -1.812463 & 0.358433 \\
\hline
\end{tabular}




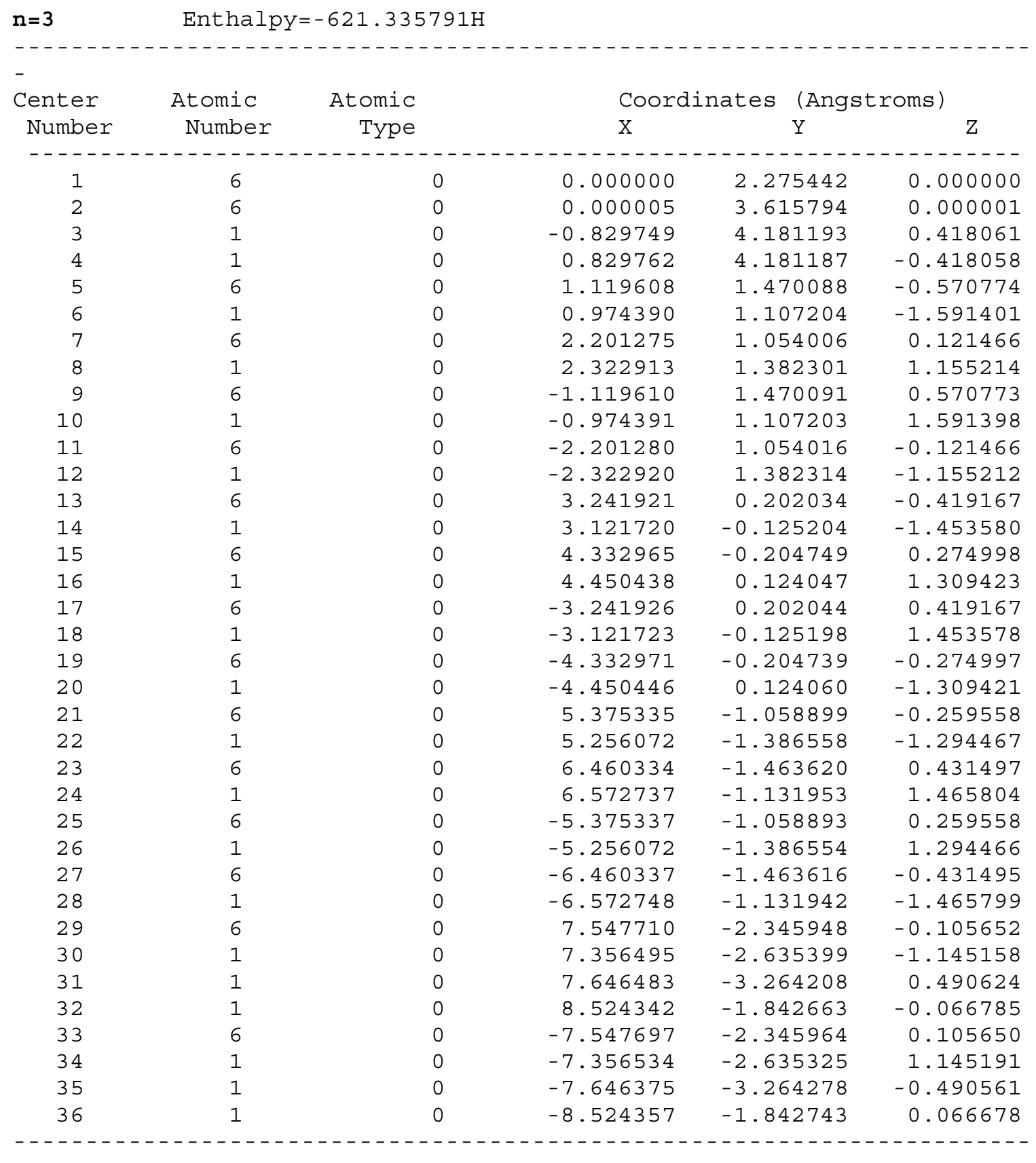




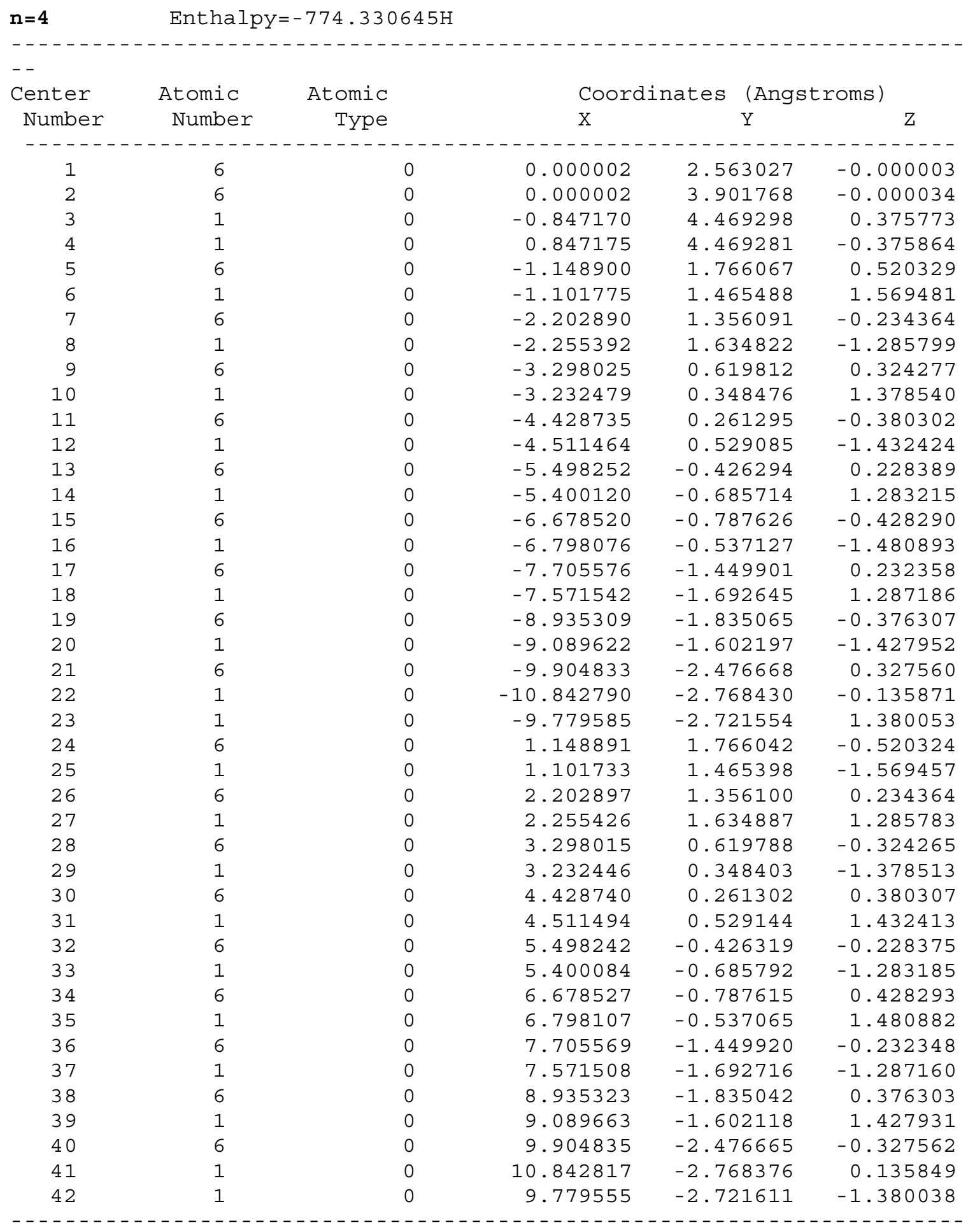




\section{Y-Shape Reference Neutral}

\begin{tabular}{|c|c|c|c|c|c|}
\hline \multicolumn{6}{|l|}{$\mathrm{n}=0$} \\
\hline \multicolumn{6}{|c|}{$\begin{array}{l}--------------------------------------------------------------------- \\
--\end{array}$} \\
\hline \multirow{2}{*}{$\begin{array}{l}\text { Center } \\
\text { Number }\end{array}$} & \multirow{2}{*}{$\begin{array}{l}\text { Atomic } \\
\text { Number }\end{array}$} & \multirow{2}{*}{$\begin{array}{l}\text { Atomic } \\
\text { Type }\end{array}$} & \multicolumn{3}{|c|}{ Coordinates (Angstroms) } \\
\hline & & & $\mathrm{X}$ & $\mathrm{Y}$ & $\mathrm{Z}$ \\
\hline----- & 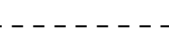 & -5 & -------- & --------- & -------- \\
\hline 1 & 6 & 0 & 0.000000 & 0.000000 & 0.373144 \\
\hline 2 & 1 & 0 & 0.000000 & 0.000000 & 1.474220 \\
\hline 3 & 6 & 0 & 0.000000 & 1.462435 & -0.095956 \\
\hline 4 & 1 & 0 & 0.886176 & 1.998562 & 0.265570 \\
\hline 5 & 1 & 0 & -0.886176 & 1.998562 & 0.265570 \\
\hline 6 & 1 & 0 & 0.000000 & 1.522513 & -1.193099 \\
\hline 7 & 6 & 0 & -1.266506 & -0.731218 & -0.095956 \\
\hline 8 & 1 & 0 & -2.173893 & -0.231830 & 0.265570 \\
\hline 9 & 1 & 0 & -1.287718 & -1.766732 & 0.265570 \\
\hline 10 & 1 & 0 & -1.318535 & -0.761256 & -1.193099 \\
\hline 11 & 6 & 0 & 1.266506 & -0.731218 & -0.095956 \\
\hline 12 & 1 & 0 & 2.173893 & -0.231830 & 0.265570 \\
\hline 13 & 1 & 0 & 1.318535 & -0.761256 & -1.193099 \\
\hline 14 & 1 & 0 & 1.287718 & -1.766732 & 0.265570 \\
\hline
\end{tabular}

\begin{tabular}{|c|c|c|c|c|c|}
\hline \multicolumn{6}{|c|}{$\mathbf{n}=1 \quad$ Enthalpy $=-313.053022 \mathrm{H}$} \\
\hline \multicolumn{6}{|c|}{ 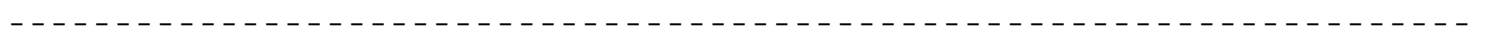 } \\
\hline \multirow{2}{*}{$\begin{array}{l}\text { Center } \\
\text { Number }\end{array}$} & \multirow{2}{*}{$\begin{array}{l}\text { Atomic } \\
\text { Number }\end{array}$} & \multirow{2}{*}{$\begin{array}{l}\text { Atomic } \\
\text { Type }\end{array}$} & \multicolumn{3}{|c|}{ Coordinates (Angstroms) } \\
\hline & & & $\mathrm{X}$ & $\mathrm{Y}$ & Z \\
\hline-----1 & 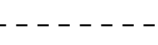 & & -------- & ------- & -------- \\
\hline 1 & 6 & 0 & -0.000025 & 0.768196 & -0.256566 \\
\hline 2 & 1 & 0 & -0.000006 & 0.621953 & -1.345681 \\
\hline 3 & 6 & 0 & 0.000837 & 2.288170 & 0.029177 \\
\hline 4 & 1 & 0 & -0.889653 & 2.764110 & -0.397892 \\
\hline 5 & 1 & 0 & 0.891750 & 2.763116 & -0.398095 \\
\hline 6 & 1 & 0 & 0.001083 & 2.484245 & 1.109413 \\
\hline 7 & 6 & 0 & 1.244869 & 0.119340 & 0.310367 \\
\hline 8 & 1 & 0 & 1.359418 & 0.184114 & 1.396220 \\
\hline 9 & 6 & 0 & 2.188172 & -0.503940 & -0.404996 \\
\hline 10 & 1 & 0 & 2.066435 & -0.567937 & -1.488928 \\
\hline 11 & 6 & 0 & -1.245783 & 0.120801 & 0.310132 \\
\hline 12 & 1 & 0 & -1.362074 & 0.188560 & 1.395622 \\
\hline 13 & 6 & 0 & -2.187816 & -0.504652 & -0.405001 \\
\hline 14 & 1 & 0 & -2.064389 & -0.571526 & -1.488567 \\
\hline 15 & 6 & 0 & 3.427383 & -1.142642 & 0.159229 \\
\hline 16 & 1 & 0 & 3.474330 & -1.035397 & 1.248990 \\
\hline 17 & 1 & 0 & 4.336025 & -0.693762 & -0.265534 \\
\hline 18 & 1 & 0 & 3.465024 & -2.214998 & -0.078103 \\
\hline 19 & 6 & 0 & -3.427775 & -1.142092 & 0.159003 \\
\hline 20 & 1 & 0 & -3.476249 & -1.032296 & 1.248443 \\
\hline 21 & 1 & 0 & -3.465020 & -2.215000 & -0.075876 \\
\hline 22 & 1 & 0 & -4.335850 & -0.694263 & -0.268079 \\
\hline
\end{tabular}




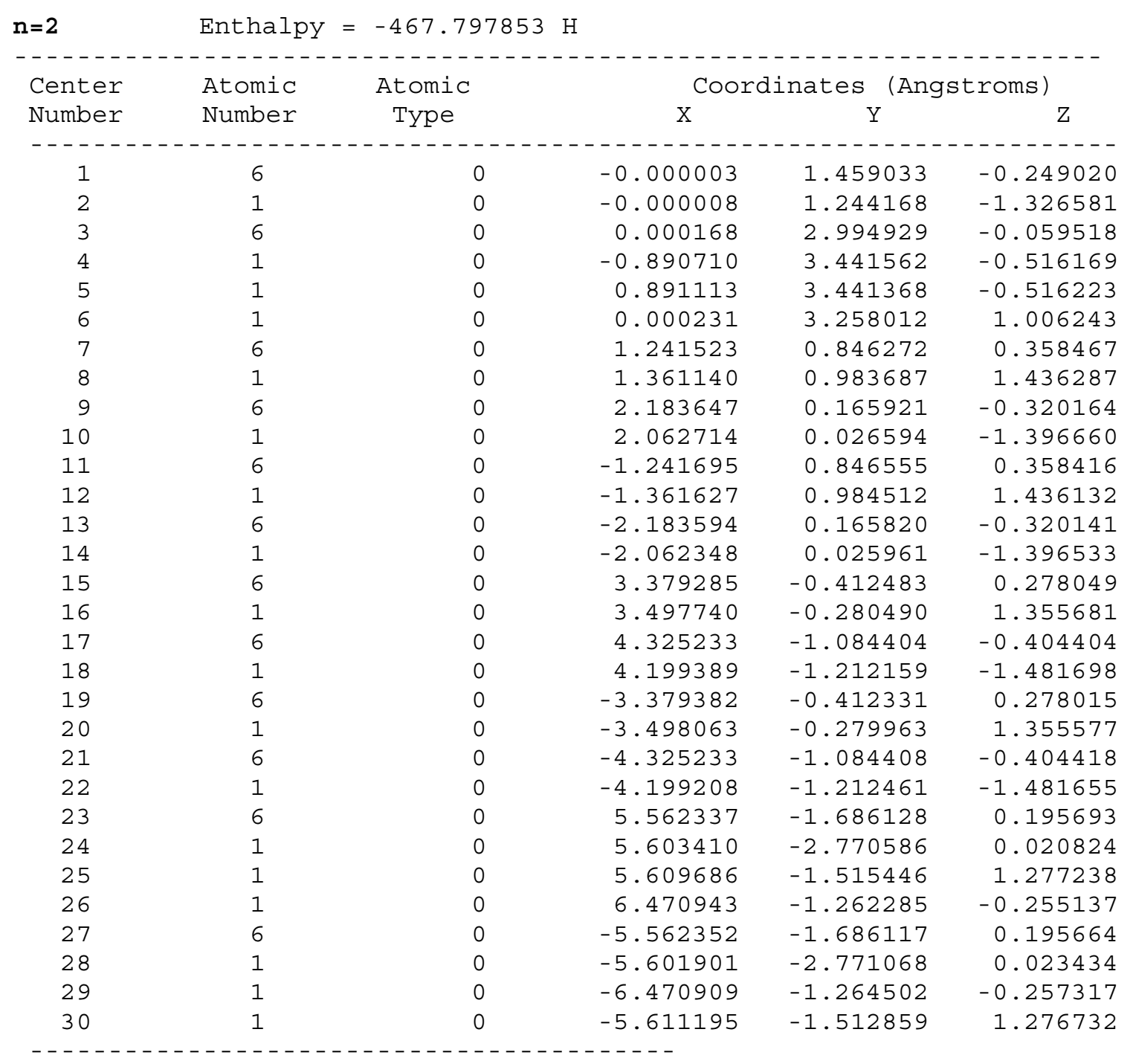




\begin{tabular}{|c|c|c|c|c|c|}
\hline$n=3$ & Enthalp & -622.545 & & & \\
\hline------- & $-\ldots \ldots$ & 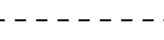 & -------- & --------- & --------- \\
\hline Center & Atomic & Atomic & Coor & nates (Ang & Eroms ) \\
\hline Number & Number & Type & $\mathrm{X}$ & $\mathrm{Y}$ & Z \\
\hline------ & ------ & ------ & --------- & -------- & ---------- \\
\hline 1 & 6 & 0 & 0.000000 & 2.128857 & -0.264435 \\
\hline 2 & 1 & 0 & 0.000026 & 1.876633 & -1.333823 \\
\hline 3 & 6 & 0 & 0.000416 & 3.670557 & -0.127343 \\
\hline 4 & 1 & 0 & -0.890378 & 4.101357 & -0.599037 \\
\hline 5 & 1 & 0 & 0.891401 & 4.100870 & -0.599108 \\
\hline 6 & 1 & 0 & 0.000546 & 3.969184 & 0.928951 \\
\hline 7 & 6 & 0 & 1.240098 & 1.536550 & 0.364443 \\
\hline 8 & 1 & 0 & 1.365387 & 1.719304 & 1.434658 \\
\hline 9 & 6 & 0 & 2.177078 & 0.820987 & -0.289606 \\
\hline 10 & 1 & 0 & 2.048545 & 0.637283 & -1.358490 \\
\hline 11 & 6 & 0 & -1.240528 & 1.537238 & 0.364236 \\
\hline 12 & 1 & 0 & -1.366561 & 1.721201 & 1.434159 \\
\hline 13 & 6 & 0 & -2.177012 & 0.820849 & -0.289616 \\
\hline 14 & 1 & 0 & -2.047758 & 0.635925 & -1.358202 \\
\hline 15 & 6 & 0 & 3.368437 & 0.266768 & 0.323160 \\
\hline 16 & 1 & 0 & 3.498028 & 0.442058 & 1.392824 \\
\hline 17 & 6 & 0 & 4.314103 & -0.440556 & -0.342464 \\
\hline 18 & 1 & 0 & 4.183887 & -0.615196 & -1.412285 \\
\hline 19 & 6 & 0 & -3.368753 & 0.267281 & 0.322993 \\
\hline 20 & 1 & 0 & -3.499130 & 0.443914 & 1.392340 \\
\hline 21 & 6 & 0 & -4.313879 & -0.440967 & -0.342415 \\
\hline 22 & 1 & 0 & -4.182866 & -0.616979 & -1.411913 \\
\hline 23 & 6 & 0 & 5.507839 & -0.993088 & 0.267444 \\
\hline 24 & 1 & 0 & 5.634751 & -0.818432 & 1.338032 \\
\hline 25 & 6 & 0 & 6.451034 & -1.693952 & -0.394095 \\
\hline 26 & 1 & 0 & 6.318324 & -1.865012 & -1.464379 \\
\hline 27 & 6 & 0 & -5.508029 & -0.992788 & 0.267327 \\
\hline 28 & 1 & 0 & -5.635722 & -0.816785 & 1.337601 \\
\hline 29 & 6 & 0 & -6.450717 & -1.694526 & -0.394008 \\
\hline 30 & 1 & 0 & -6.317209 & -1.866966 & -1.463972 \\
\hline 31 & 6 & 0 & 7.690966 & -2.270413 & 0.222877 \\
\hline 32 & 1 & 0 & 8.596990 & -1.865961 & -0.250619 \\
\hline 33 & 1 & 0 & 7.731324 & -3.361233 & 0.092197 \\
\hline 34 & 1 & 0 & 7.744996 & -2.055580 & 1.296161 \\
\hline 35 & 6 & 0 & -7.691122 & -2.270168 & 0.222775 \\
\hline 36 & 1 & 0 & -8.596789 & -1.865843 & -0.251524 \\
\hline 37 & 1 & 0 & -7.745668 & -2.054449 & 1.295855 \\
\hline 38 & 1 & 0 & -7.731713 & -3.361076 & 0.092942 \\
\hline
\end{tabular}




\begin{tabular}{|c|c|c|c|c|c|}
\hline $\mathrm{n}=4$ & Enthal & $=-777$. & $59 \mathrm{H}$ & & \\
\hline------- & 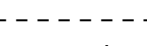 & -------- & ---------- & ---------- & -------- \\
\hline Center & Atomic & Atomic & Coor & inates (Ans & roms ) \\
\hline Number & Number & Type & $\mathrm{X}$ & $\mathrm{Y}$ & Z \\
\hline------ & 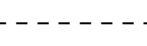 & 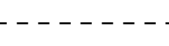 & ---------- & --------- & --------- \\
\hline 1 & 6 & 0 & -0.000090 & 2.780887 & -0.284725 \\
\hline 2 & 6 & 0 & -0.000920 & 4.325327 & -0.183812 \\
\hline 3 & 1 & 0 & 0.889687 & 4.745145 & -0.665580 \\
\hline 4 & 1 & 0 & -0.001037 & 4.648297 & 0.865237 \\
\hline 5 & 1 & 0 & -0.892044 & 4.744169 & -0.665461 \\
\hline 6 & 1 & 0 & -0.000302 & 2.503084 & -1.347706 \\
\hline 7 & 6 & 0 & -1.239487 & 2.203078 & 0.358340 \\
\hline 8 & 1 & 0 & -1.368602 & 2.416350 & 1.422354 \\
\hline 9 & 6 & 0 & -2.172843 & 1.465342 & -0.278105 \\
\hline 10 & 1 & 0 & -2.039527 & 1.251138 & -1.340683 \\
\hline 11 & 6 & 0 & -3.364197 & 0.928459 & 0.344582 \\
\hline 12 & 1 & 0 & -3.501154 & 1.135602 & 1.407497 \\
\hline 13 & 6 & 0 & -4.307318 & 0.198812 & -0.306899 \\
\hline 14 & 1 & 0 & -4.167534 & -0.006891 & -1.369973 \\
\hline 15 & 6 & 0 & -5.499187 & -0.332243 & 0.306492 \\
\hline 16 & 1 & 0 & -5.640083 & -0.126578 & 1.369359 \\
\hline 17 & 6 & 0 & -6.442522 & -1.058795 & -0.347957 \\
\hline 18 & 1 & 0 & -6.302046 & -1.264789 & -1.410739 \\
\hline 19 & 6 & 0 & -7.641614 & -1.588904 & 0.266197 \\
\hline 20 & 1 & 0 & -7.778978 & -1.381911 & 1.329669 \\
\hline 21 & 6 & 0 & -8.580903 & -2.307519 & -0.383686 \\
\hline 22 & 1 & 0 & -8.437834 & -2.510883 & -1.446954 \\
\hline 23 & 6 & 0 & -9.828381 & -2.861557 & 0.237617 \\
\hline 24 & 1 & 0 & -9.871703 & -3.955499 & 0.137365 \\
\hline 25 & 1 & 0 & -10.728238 & -2.467765 & -0.256268 \\
\hline 26 & 1 & 0 & -9.892008 & -2.615990 & 1.303698 \\
\hline 27 & 6 & 0 & 1.240422 & 2.204527 & 0.357480 \\
\hline 28 & 1 & 0 & 1.371260 & 2.420101 & 1.420822 \\
\hline 29 & 6 & 0 & 2.172769 & 1.465385 & -0.278807 \\
\hline 30 & 1 & 0 & 2.037782 & 1.248766 & -1.340695 \\
\hline 31 & 6 & 0 & 3.365077 & 0.929961 & 0.343298 \\
\hline 32 & 1 & 0 & 3.503721 & 1.139906 & 1.405434 \\
\hline 33 & 6 & 0 & 4.307084 & 0.198376 & -0.307585 \\
\hline 34 & 1 & 0 & 4.165702 & -0.010219 & -1.369837 \\
\hline 35 & 6 & 0 & 5.499750 & -0.331186 & 0.305604 \\
\hline 36 & 1 & 0 & 5.642210 & -0.122541 & 1.367678 \\
\hline 37 & 6 & 0 & 6.442056 & -1.059696 & -0.348184 \\
\hline 38 & 1 & 0 & 6.300043 & -1.268760 & -1.410198 \\
\hline 39 & 6 & 0 & 7.641979 & -1.588203 & 0.265747 \\
\hline 40 & 1 & 0 & 7.780803 & -1.377859 & 1.328378 \\
\hline 41 & 6 & 0 & 8.580233 & -2.309008 & -0.383154 \\
\hline 42 & 1 & 0 & 8.435755 & -2.515704 & -1.445577 \\
\hline 43 & 6 & 0 & 9.828384 & -2.861346 & 0.238361 \\
\hline 44 & 1 & 0 & 9.870560 & -3.955775 & 0.143008 \\
\hline 45 & 1 & 0 & 9.894129 & -2.611061 & 1.303225 \\
\hline 46 & 1 & 0 & 10.727684 & -2.470529 & -0.258873 \\
\hline
\end{tabular}




\section{Y-Shape Reference Dication}

\begin{tabular}{|c|c|c|c|c|c|}
\hline \multicolumn{6}{|c|}{ Enthalpy $=-156.216482 \mathrm{H}$} \\
\hline \multicolumn{6}{|c|}{ 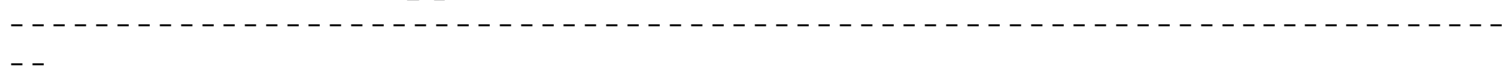 } \\
\hline \multirow{2}{*}{$\begin{array}{l}\text { Center } \\
\text { Number }\end{array}$} & \multirow{2}{*}{$\begin{array}{l}\text { Atomic } \\
\text { Number }\end{array}$} & \multirow{2}{*}{$\begin{array}{l}\text { Atomic } \\
\text { Type }\end{array}$} & \multicolumn{3}{|c|}{ Coordinates (Angstroms) } \\
\hline & & & $\mathrm{X}$ & $\mathrm{Y}$ & Z \\
\hline----- & 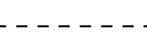 & ----- & -------1 & -------1 & -------- \\
\hline 1 & 6 & 0 & -0.011168 & -0.327598 & -0.480572 \\
\hline 2 & 1 & 0 & -0.006222 & -0.165852 & -1.563008 \\
\hline 3 & 6 & 0 & -1.257838 & -0.664659 & 0.131578 \\
\hline 4 & 1 & 0 & -1.332224 & -1.006928 & 1.170225 \\
\hline 5 & 1 & 0 & -2.192079 & -0.566169 & -0.433507 \\
\hline 6 & 6 & 0 & 0.047581 & 1.467895 & 0.142629 \\
\hline 7 & 1 & 0 & -0.834773 & 1.863769 & -0.372371 \\
\hline 8 & 1 & 0 & 0.987783 & 1.792449 & -0.317779 \\
\hline 9 & 1 & 0 & 0.017318 & 1.466866 & 1.231793 \\
\hline 10 & 6 & 0 & 1.212118 & -0.741567 & 0.131209 \\
\hline 11 & 1 & 0 & 2.149757 & -0.705064 & -0.435643 \\
\hline 12 & 1 & 0 & 1.266280 & -1.083493 & 1.171224 \\
\hline
\end{tabular}

\begin{tabular}{|c|c|c|c|c|c|}
\hline $\mathrm{n}=1$ & Enthalp & -311.119 & & & \\
\hline-------- & ------ & ------- & --------- & --------- & -------- \\
\hline Center & Atomic & Atomic & Coor & inates (Ang & (roms) \\
\hline Number & Number & Type & $\mathrm{X}$ & $\mathrm{Y}$ & Z \\
\hline------ & 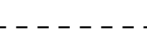 & $----\ldots$ & -------- & --------1 & --------- \\
\hline 1 & 6 & 0 & 0.000002 & 0.395311 & 0.266123 \\
\hline 2 & 1 & 0 & 0.000006 & 0.432647 & 1.360229 \\
\hline 3 & 6 & 0 & -0.000030 & 1.934576 & -0.248597 \\
\hline 4 & 1 & 0 & 0.890654 & 2.421882 & 0.157270 \\
\hline 5 & 1 & 0 & -0.890713 & 2.421847 & 0.157312 \\
\hline 6 & 1 & 0 & -0.000052 & 1.994794 & -1.339132 \\
\hline 7 & 6 & 0 & -1.251964 & -0.198107 & -0.267143 \\
\hline 8 & 1 & 0 & -1.259729 & -0.517776 & -1.312075 \\
\hline 9 & 6 & 0 & -2.429841 & -0.309099 & 0.466098 \\
\hline 10 & 1 & 0 & -2.463521 & -0.005294 & 1.510693 \\
\hline 11 & 6 & 0 & 1.251984 & -0.198061 & -0.267156 \\
\hline 12 & 1 & 0 & 1.259754 & -0.517719 & -1.312092 \\
\hline 13 & 6 & 0 & 2.429853 & -0.309077 & 0.466094 \\
\hline 14 & 1 & 0 & 2.463533 & -0.005270 & 1.510689 \\
\hline 15 & 6 & 0 & -3.562194 & -0.828455 & -0.140204 \\
\hline 16 & 1 & 0 & -4.494755 & -0.943448 & 0.411626 \\
\hline 17 & 1 & 0 & -3.564459 & -1.144982 & -1.183127 \\
\hline 18 & 6 & 0 & 3.562191 & -0.828487 & -0.140191 \\
\hline 19 & 1 & 0 & 3.564497 & -1.144880 & -1.183154 \\
\hline 20 & 1 & 0 & 4.494773 & -0.943407 & 0.411619 \\
\hline
\end{tabular}




\begin{tabular}{|c|c|c|c|c|c|}
\hline \multicolumn{6}{|c|}{$\mathbf{n}=2 \quad$ Enthalpy $=-465.955450 \mathrm{H}$} \\
\hline \multicolumn{6}{|c|}{ 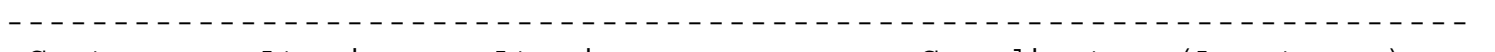 } \\
\hline \multirow{2}{*}{$\begin{array}{l}\text { Center } \\
\text { Number }\end{array}$} & \multirow{2}{*}{$\begin{array}{l}\text { Atomic } \\
\text { Number }\end{array}$} & \multirow{2}{*}{$\begin{array}{l}\text { Atomic } \\
\text { Type }\end{array}$} & \multicolumn{3}{|c|}{ Coordinates (Angstroms) } \\
\hline & & & $\mathrm{X}$ & $\mathrm{Y}$ & Z \\
\hline \multicolumn{6}{|c|}{-----------------------------------------------------} \\
\hline 1 & 6 & 0 & -0.000003 & 0.966171 & 0.224573 \\
\hline 2 & 1 & 0 & -0.000013 & 0.881029 & 1.316376 \\
\hline 3 & 6 & 0 & 0.000060 & 2.506325 & -0.142000 \\
\hline 4 & 1 & 0 & 0.888782 & 2.976846 & 0.287834 \\
\hline 5 & 1 & 0 & -0.888666 & 2.976900 & 0.287767 \\
\hline 6 & 1 & 0 & 0.000104 & 2.662308 & -1.224626 \\
\hline 7 & 6 & 0 & -1.248301 & 0.365136 & -0.345948 \\
\hline 8 & 1 & 0 & -1.279668 & 0.225348 & -1.428353 \\
\hline 9 & 6 & 0 & -2.360821 & 0.054844 & 0.390695 \\
\hline 10 & 1 & 0 & -2.357291 & 0.183145 & 1.471831 \\
\hline 11 & 6 & 0 & 1.248257 & 0.365033 & -0.345927 \\
\hline 12 & 1 & 0 & 1.279548 & 0.225017 & -1.428306 \\
\hline 13 & 6 & 0 & 2.360833 & 0.054892 & 0.390696 \\
\hline 14 & 1 & 0 & 2.357380 & 0.183400 & 1.471808 \\
\hline 15 & 6 & 0 & -3.537285 & -0.423089 & -0.235205 \\
\hline 16 & 1 & 0 & -3.527821 & -0.546601 & -1.319076 \\
\hline 17 & 6 & 0 & -4.724916 & -0.745533 & 0.453787 \\
\hline 18 & 1 & 0 & -4.764287 & -0.632670 & 1.534958 \\
\hline 19 & 6 & 0 & 3.537254 & -0.423152 & -0.235198 \\
\hline 20 & 1 & 0 & 3.527708 & -0.546885 & -1.319044 \\
\hline 21 & 6 & 0 & 4.724937 & -0.745458 & 0.453769 \\
\hline 22 & 1 & 0 & 4.764389 & -0.632378 & 1.534914 \\
\hline 23 & 6 & 0 & -5.824083 & -1.191712 & -0.231706 \\
\hline 24 & 1 & 0 & -6.749279 & -1.440037 & 0.282968 \\
\hline 25 & 1 & 0 & -5.815170 & -1.312672 & -1.313452 \\
\hline 26 & 6 & 0 & 5.824053 & -1.191771 & -0.231718 \\
\hline 27 & 1 & 0 & 5.815073 & -1.312910 & -1.313443 \\
\hline 28 & 1 & 0 & 6.749301 & -1.439951 & 0.282933 \\
\hline & & & ---- & --------- & --------- \\
\hline
\end{tabular}




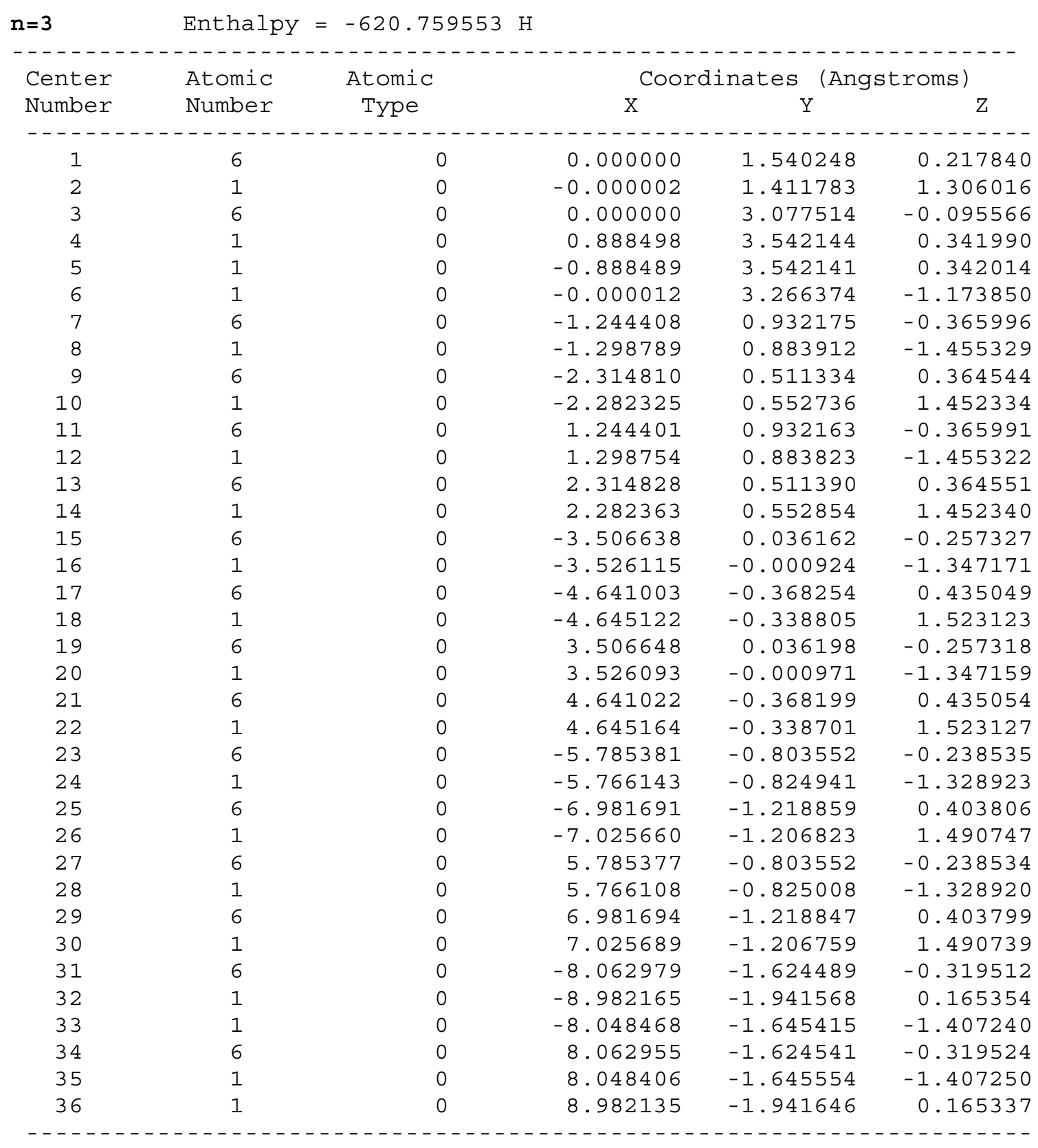




\begin{tabular}{|c|c|c|c|c|c|}
\hline \multicolumn{6}{|c|}{$\mathbf{n}=4 \quad$ Enthalpy $=-775.547976 \mathrm{H}$} \\
\hline \multicolumn{6}{|c|}{ 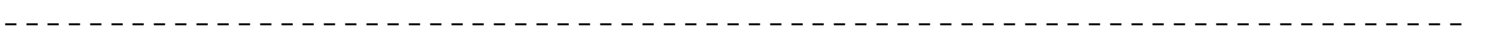 } \\
\hline \multirow{2}{*}{$\begin{array}{l}\text { Center } \\
\text { Number }\end{array}$} & \multirow{2}{*}{$\begin{array}{l}\text { Atomic } \\
\text { Number }\end{array}$} & \multirow{2}{*}{$\begin{array}{l}\text { Atomic } \\
\text { Type }\end{array}$} & \multicolumn{3}{|c|}{ Coordinates (Angstroms) } \\
\hline & & & $\mathrm{X}$ & $\mathrm{Y}$ & Z \\
\hline \multicolumn{6}{|c|}{-----------------------------------------------------} \\
\hline 1 & 6 & 0 & -0.000027 & 2.092337 & 0.195019 \\
\hline 2 & 6 & 0 & 0.000032 & 3.623378 & -0.115831 \\
\hline 3 & 1 & 0 & -0.888957 & 4.091701 & 0.317483 \\
\hline 4 & 1 & 0 & 0.000917 & 3.811473 & -1.194785 \\
\hline 5 & 1 & 0 & 0.888212 & 4.091909 & 0.318921 \\
\hline 6 & 1 & 0 & -0.000157 & 1.960280 & 1.283271 \\
\hline 7 & 6 & 0 & 1.241925 & 1.470729 & -0.385098 \\
\hline 8 & 1 & 0 & 1.309955 & 1.450229 & -1.474578 \\
\hline 9 & 6 & 0 & 2.287787 & 1.003311 & 0.346096 \\
\hline 10 & 1 & 0 & 2.238657 & 1.019982 & 1.434299 \\
\hline 11 & 6 & 0 & 3.485120 & 0.511197 & -0.263252 \\
\hline 12 & 1 & 0 & 3.522187 & 0.496160 & -1.353119 \\
\hline 13 & 6 & 0 & 4.591363 & 0.073661 & 0.437781 \\
\hline 14 & 1 & 0 & 4.573621 & 0.083715 & 1.526358 \\
\hline 15 & 6 & 0 & 5.755487 & -0.374776 & -0.215167 \\
\hline 16 & 1 & 0 & 5.757339 & -0.376639 & -1.305839 \\
\hline 17 & 6 & 0 & 6.911175 & -0.810818 & 0.442063 \\
\hline 18 & 1 & 0 & 6.931370 & -0.816981 & 1.530468 \\
\hline 19 & 6 & 0 & 8.033661 & -1.228954 & -0.259349 \\
\hline 20 & 1 & 0 & 7.998726 & -1.215759 & -1.349372 \\
\hline 21 & 6 & 0 & 9.242324 & -1.676338 & 0.350579 \\
\hline 22 & 1 & 0 & 9.297987 & -1.697294 & 1.437079 \\
\hline 23 & 6 & 0 & 10.309745 & -2.068714 & -0.392536 \\
\hline 24 & 1 & 0 & 11.230562 & -2.407924 & 0.072754 \\
\hline 25 & 1 & 0 & 10.283880 & -2.057628 & -1.480019 \\
\hline 26 & 6 & 0 & -1.241874 & 1.470726 & -0.385328 \\
\hline 27 & 1 & 0 & -1.309751 & 1.450136 & -1.474790 \\
\hline 28 & 6 & 0 & -2.287806 & 1.003359 & 0.345786 \\
\hline 29 & 1 & 0 & -2.238790 & 1.020229 & 1.433947 \\
\hline 30 & 6 & 0 & -3.485061 & 0.511226 & -0.263701 \\
\hline 31 & 1 & 0 & -3.521894 & 0.495927 & -1.353531 \\
\hline 32 & 6 & 0 & -4.591504 & 0.074042 & 0.437210 \\
\hline 33 & 1 & 0 & -4.573930 & 0.084374 & 1.525790 \\
\hline 34 & 6 & 0 & -5.755592 & -0.374569 & -0.215675 \\
\hline 35 & 1 & 0 & -5.757473 & -0.376778 & -1.306338 \\
\hline 36 & 6 & 0 & -6.911177 & -0.810504 & 0.441792 \\
\hline 37 & 1 & 0 & -6.931251 & -0.816321 & 1.530199 \\
\hline 38 & 6 & 0 & -8.033703 & -1.229027 & -0.259324 \\
\hline 39 & 1 & 0 & -7.998902 & -1.216188 & -1.349351 \\
\hline 40 & 6 & 0 & -9.242227 & -1.676369 & 0.350896 \\
\hline 41 & 1 & 0 & -9.297769 & -1.697002 & 1.437405 \\
\hline 42 & 6 & 0 & -10.309680 & -2.069073 & -0.392001 \\
\hline 43 & 1 & 0 & -10.283921 & -2.058294 & -1.479488 \\
\hline 44 & 1 & 0 & -11.230419 & -2.408239 & 0.073475 \\
\hline
\end{tabular}




\section{Linear Neutral}

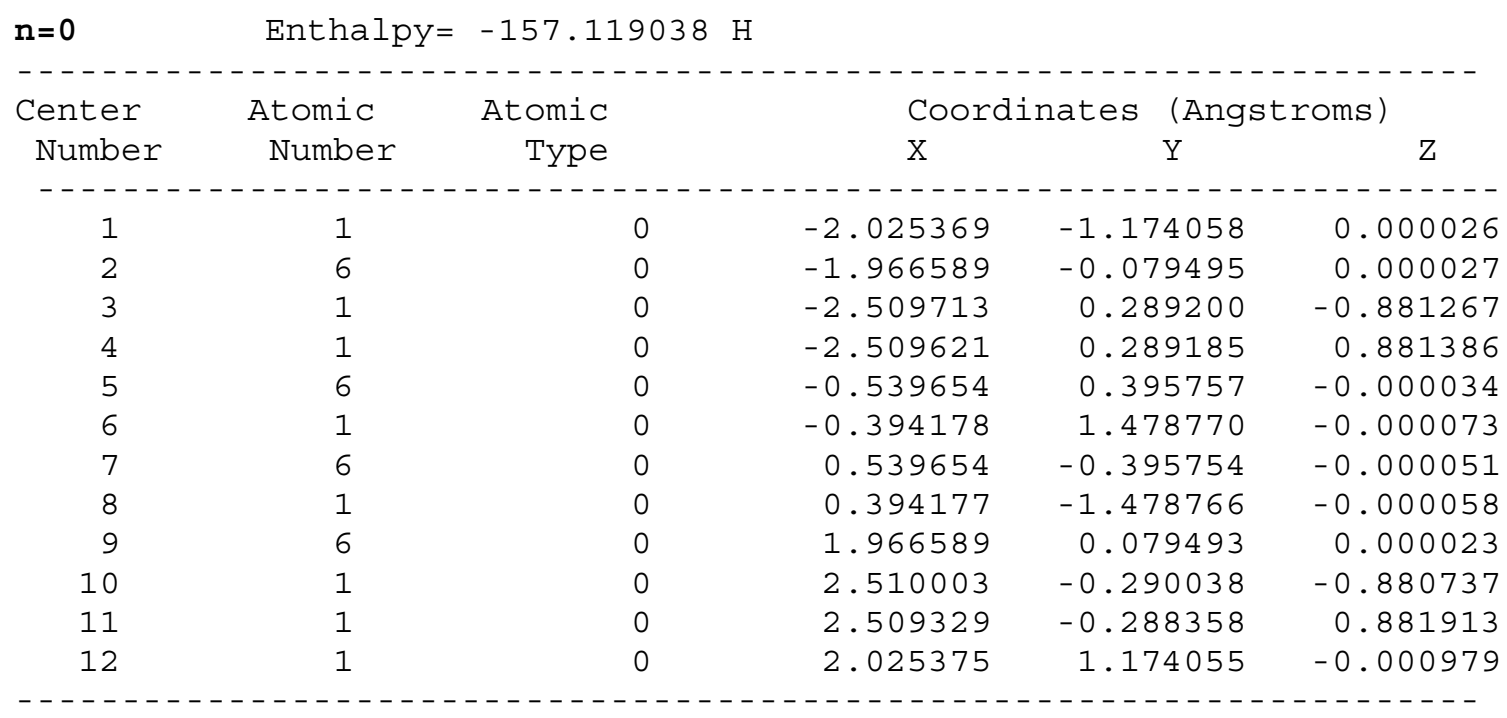

\section{Linear Dication}

\begin{tabular}{|c|c|c|c|c|c|}
\hline $\mathrm{n}=0$ & Enthalp & -155.019025 & & & \\
\hline \multirow{2}{*}{$\begin{array}{l}\text { Center } \\
\text { Number }\end{array}$} & \multirow{2}{*}{$\begin{array}{l}\text { Atomic } \\
\text { Number }\end{array}$} & \multirow{2}{*}{$\begin{array}{r}\text { Atomic } \\
\text { Type }\end{array}$} & \multicolumn{3}{|c|}{ Coordinates (Angstroms) } \\
\hline & & & $\mathrm{X}$ & $\mathrm{Y}$ & Z \\
\hline & ( & & - & 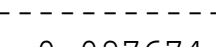 & 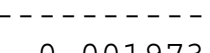 \\
\hline 1 & 6 & 0 & 1.889908 & -0.097674 & -0.001973 \\
\hline 2 & 1 & 0 & 2.707453 & 0.556171 & 0.329747 \\
\hline 3 & 1 & 0 & 2.136854 & -1.135877 & -0.254846 \\
\hline 4 & 6 & 0 & 0.548913 & 0.421735 & -0.072922 \\
\hline 5 & 1 & 0 & 0.426273 & 1.502153 & -0.193073 \\
\hline 6 & 6 & 0 & -0.548889 & -0.421848 & 0.072399 \\
\hline 7 & 1 & 0 & -0.425949 & -1.502224 & 0.192219 \\
\hline 8 & 6 & 0 & -1.889988 & 0.097769 & 0.002391 \\
\hline 9 & 1 & 0 & -2.707919 & -0.556466 & -0.327585 \\
\hline 10 & 1 & 0 & -2.136371 & 1.136352 & 0.254176 \\
\hline
\end{tabular}




\section{Linear Parallel Neutral}

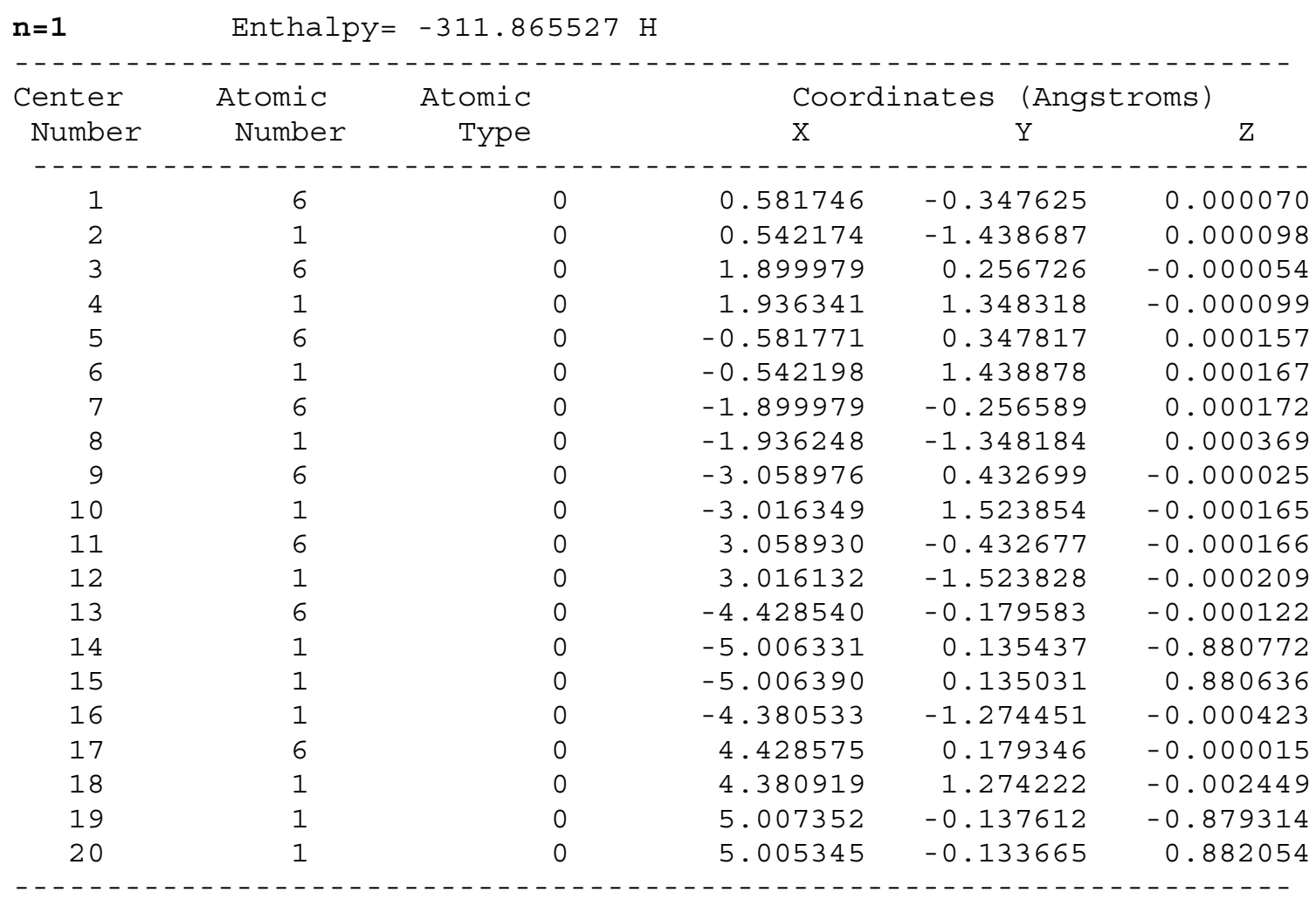




\begin{tabular}{|c|c|c|c|c|c|}
\hline \multicolumn{3}{|c|}{$\mathrm{n}=\mathbf{2} \quad$ Enthalpy $=-466.614906 \mathrm{H}$} & & & \\
\hline \multicolumn{6}{|c|}{--------------------------------------------------------------------} \\
\hline \multirow{2}{*}{$\begin{array}{l}\text { Center } \\
\text { Number }\end{array}$} & \multirow{2}{*}{$\begin{array}{l}\text { Atomic } \\
\text { Number }\end{array}$} & \multirow{2}{*}{$\begin{array}{l}\text { Atomic } \\
\text { Type }\end{array}$} & \multicolumn{3}{|c|}{ Coordinates (Angstroms) } \\
\hline & & & \multirow{2}{*}{$\mathrm{X}$} & $\mathrm{Y}$ & \multirow{2}{*}{$\begin{array}{c}\mathrm{Z} \\
------\end{array}$} \\
\hline----- & ------ & & & -------1 & \\
\hline 1 & 6 & 0 & -0.591853 & 0.337077 & -0.000005 \\
\hline 2 & 1 & 0 & -0.571628 & 1.428610 & -0.000004 \\
\hline 3 & 6 & 0 & -1.887262 & -0.289987 & -0.000009 \\
\hline 4 & 1 & 0 & -1.906598 & -1.381609 & -0.000012 \\
\hline 5 & 6 & 0 & 0.591853 & -0.337076 & -0.000005 \\
\hline 6 & 1 & 0 & 0.571629 & -1.428609 & -0.000006 \\
\hline 7 & 6 & 0 & 1.887262 & 0.289988 & -0.000005 \\
\hline 8 & 1 & 0 & 1.906599 & 1.381610 & -0.000005 \\
\hline 9 & 6 & 0 & 3.069368 & -0.382505 & -0.000004 \\
\hline 10 & 1 & 0 & 3.052044 & -1.474040 & -0.000003 \\
\hline 11 & 6 & 0 & -3.069368 & 0.382505 & -0.000007 \\
\hline 12 & 1 & 0 & -3.052045 & 1.474040 & -0.000003 \\
\hline 13 & 6 & 0 & 4.370817 & 0.249775 & -0.000003 \\
\hline 14 & 1 & 0 & 4.383689 & 1.341878 & -0.000006 \\
\hline 15 & 6 & 0 & 5.545864 & -0.414663 & -0.000002 \\
\hline 16 & 1 & 0 & 5.526885 & -1.506428 & -0.000005 \\
\hline 17 & 6 & 0 & -4.370817 & -0.249775 & -0.000010 \\
\hline 18 & 1 & 0 & -4.383688 & -1.341878 & -0.000018 \\
\hline 19 & 6 & 0 & -5.545864 & 0.414662 & -0.000007 \\
\hline 20 & 1 & 0 & -5.526886 & 1.506427 & -0.000010 \\
\hline 21 & 6 & 0 & -6.900820 & -0.227671 & 0.000022 \\
\hline 22 & 1 & 0 & -7.485699 & 0.074970 & -0.880404 \\
\hline 23 & 1 & 0 & -7.485471 & 0.074583 & 0.880738 \\
\hline 24 & 1 & 0 & -6.829198 & -1.321220 & -0.000219 \\
\hline 25 & 6 & 0 & 6.900820 & 0.227669 & 0.000016 \\
\hline 26 & 1 & 0 & 6.829198 & 1.321218 & -0.000114 \\
\hline 27 & 1 & 0 & 7.485647 & -0.074884 & -0.880476 \\
\hline 28 & 1 & 0 & 7.485521 & -0.074674 & 0.880666 \\
\hline-5 & & & --- & --------- & -------- \\
\hline
\end{tabular}




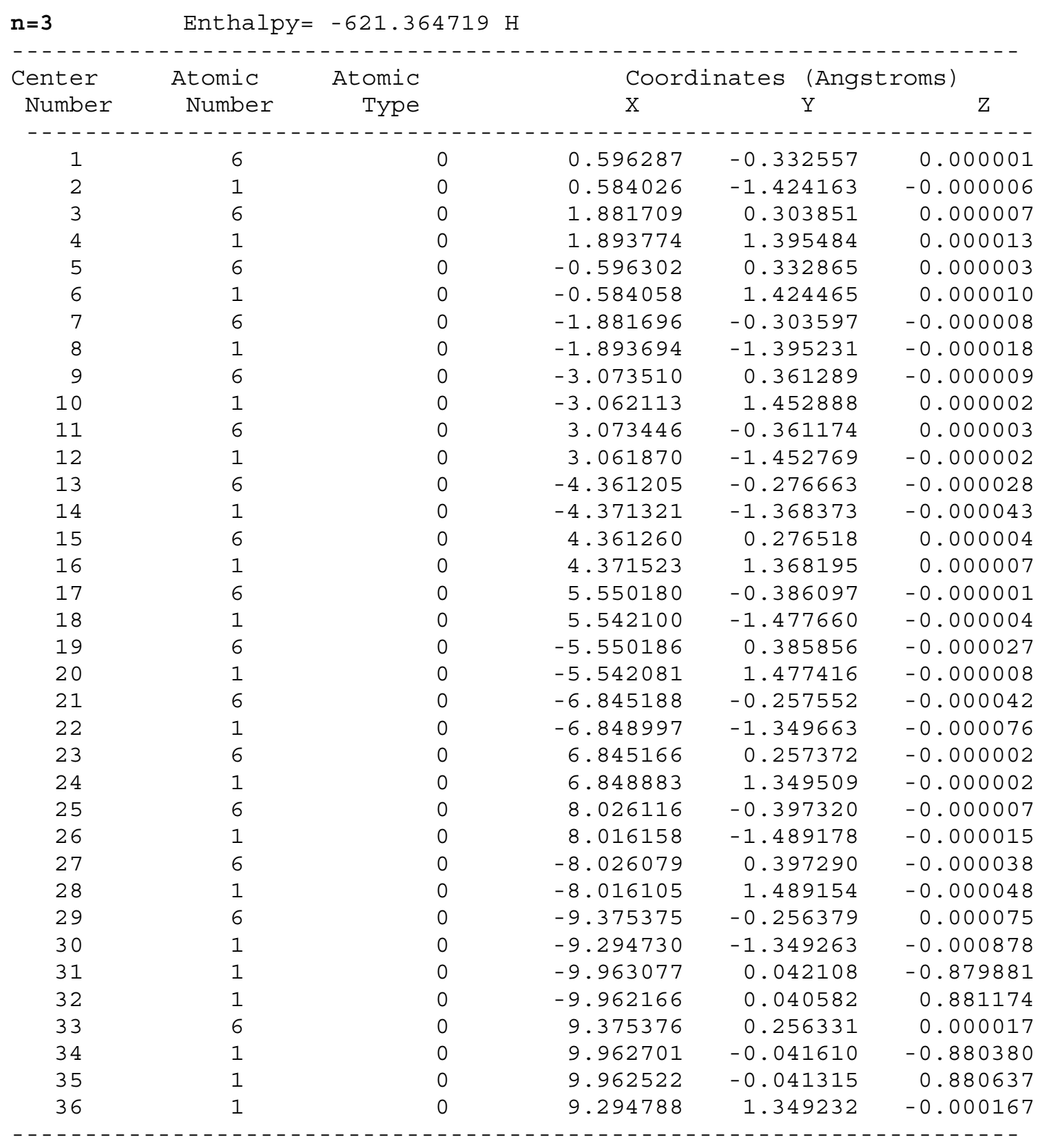




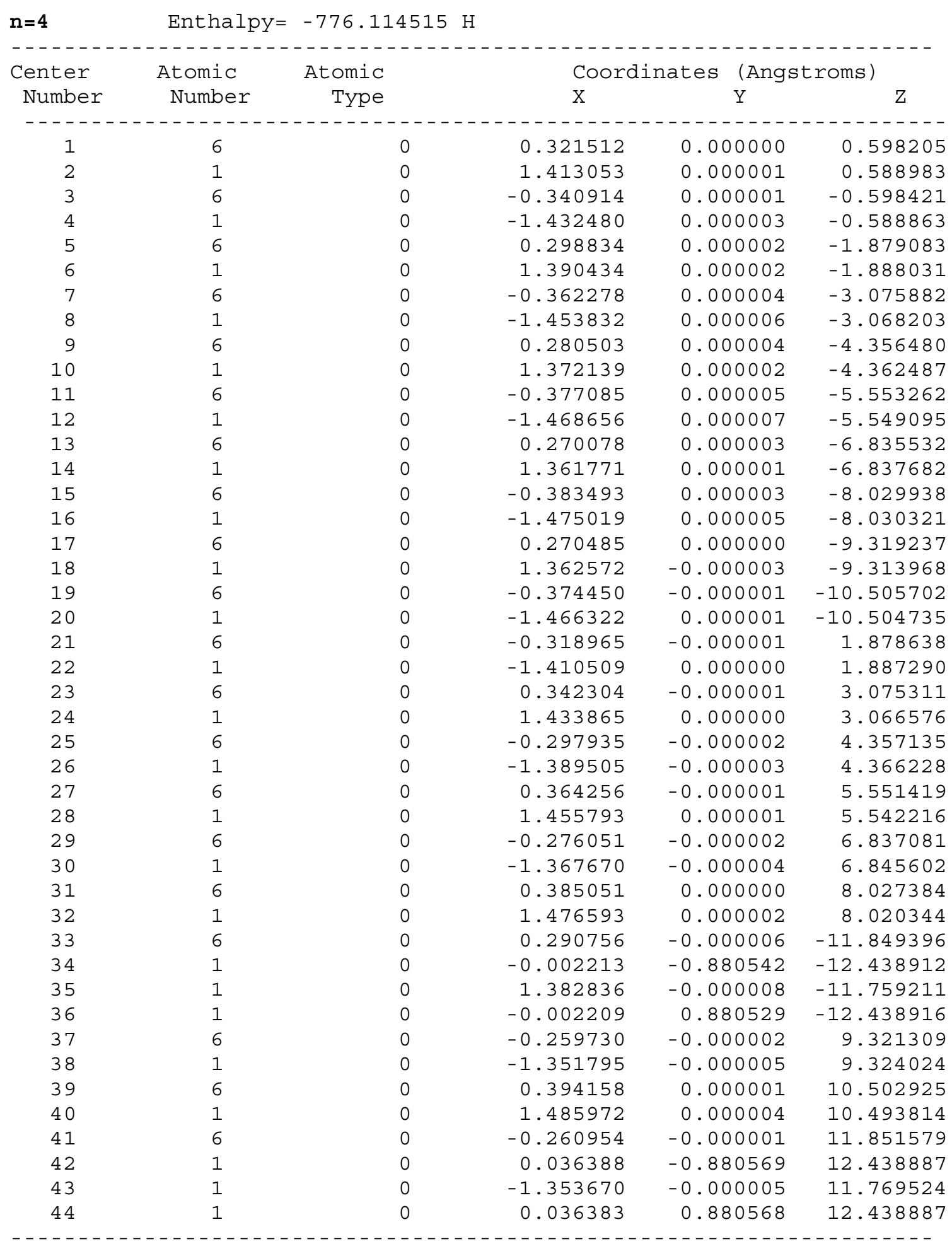




\section{Linear Parallel Dication}

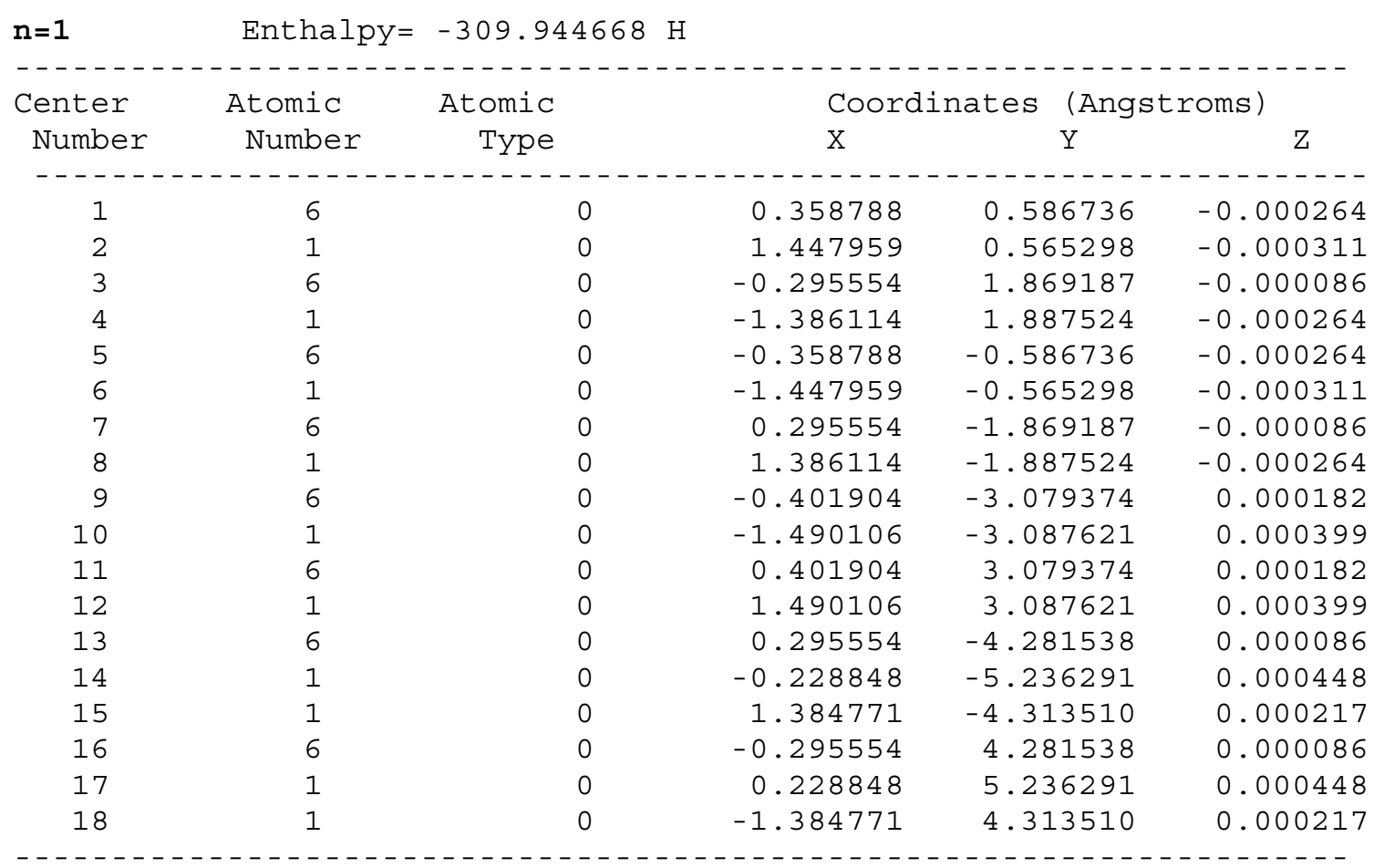




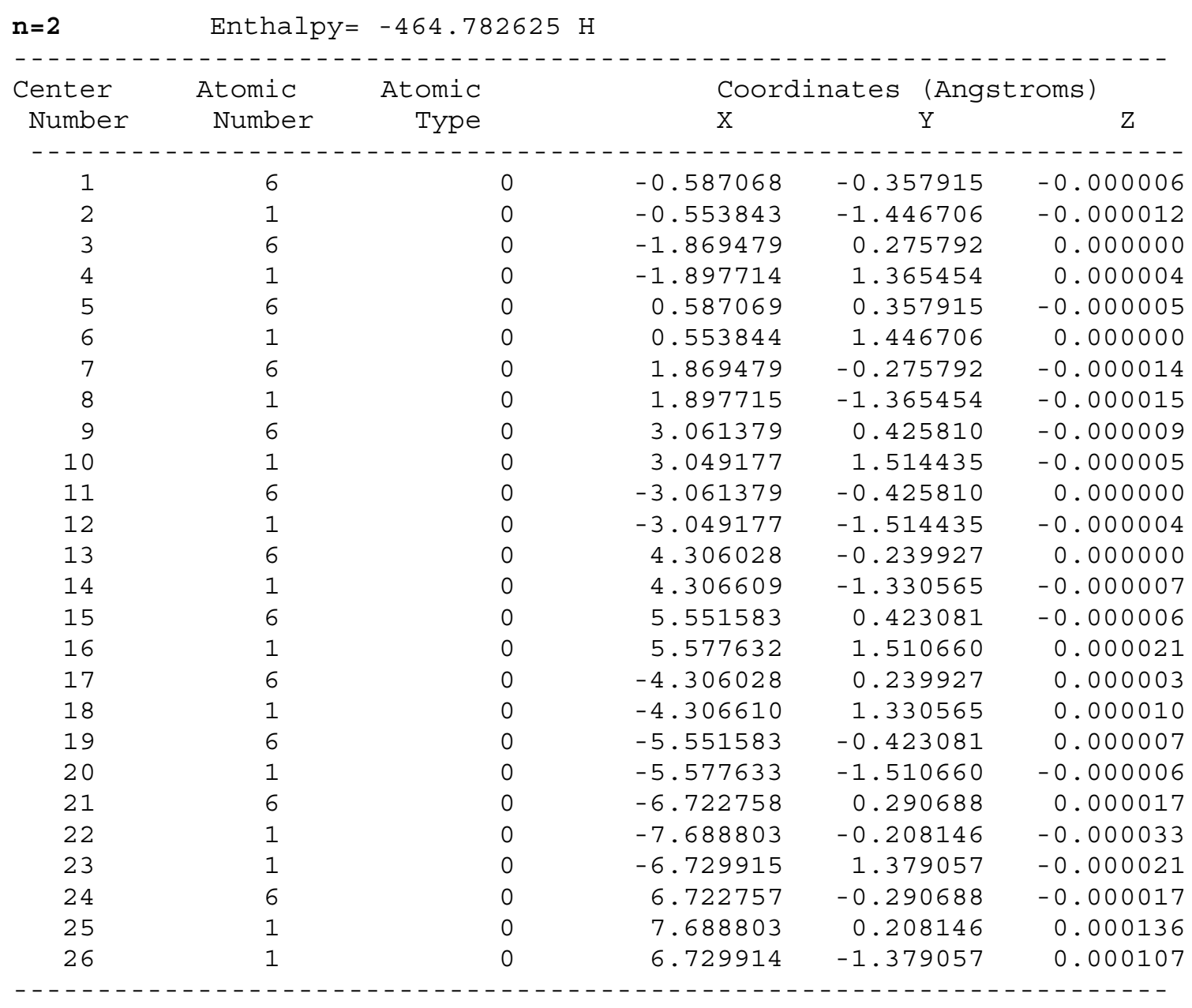




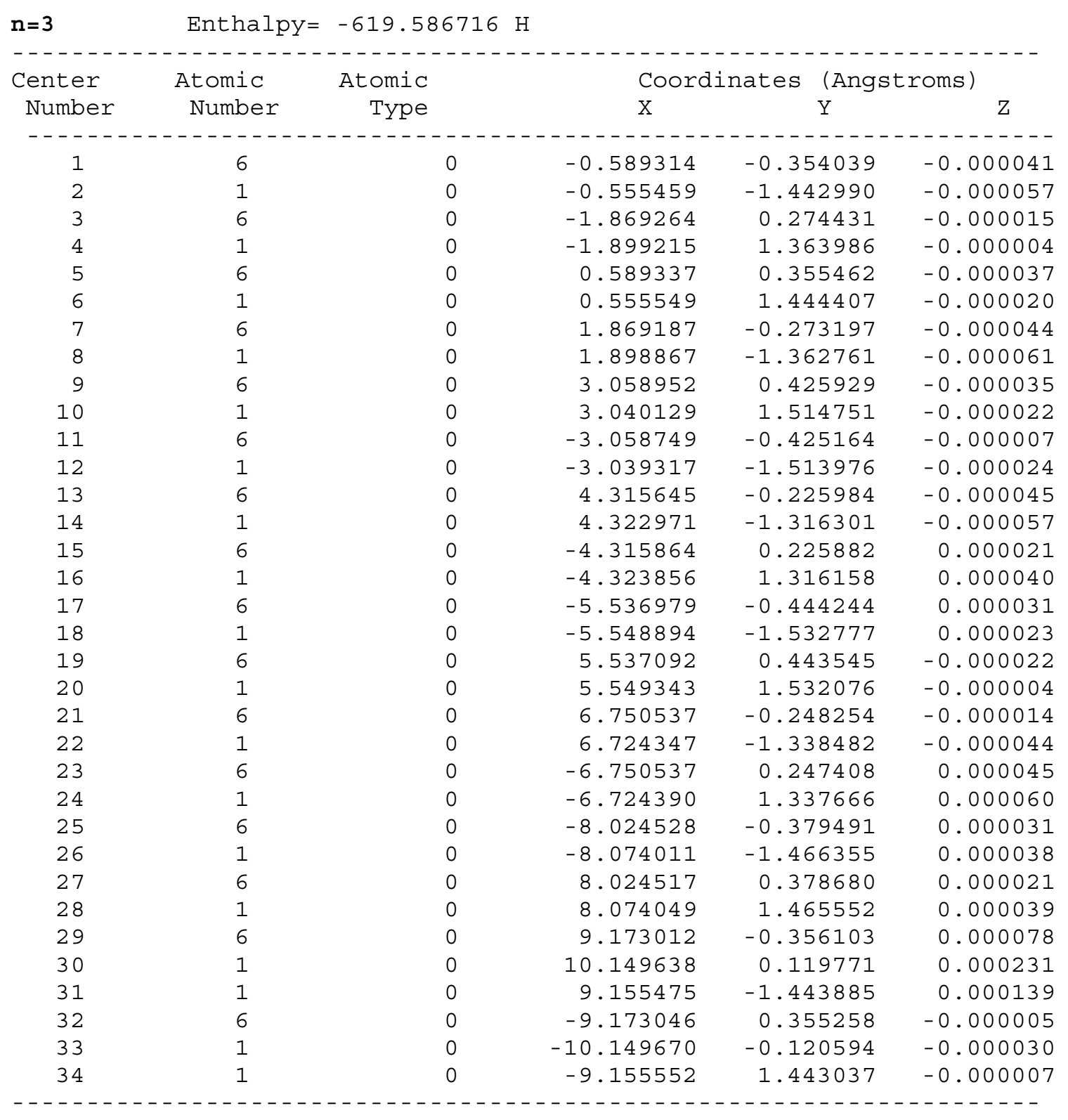




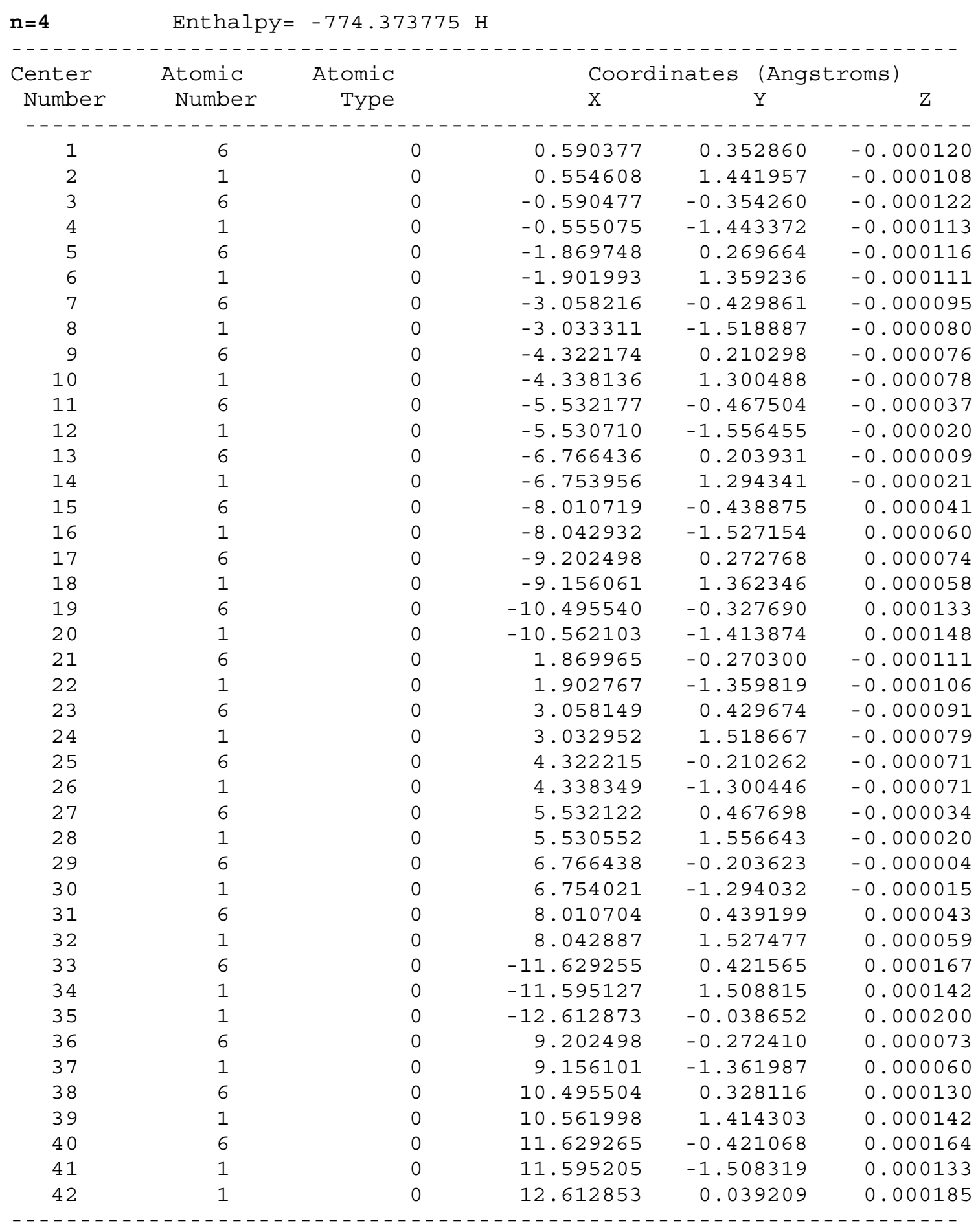




\section{Linear Perpendicular Neutral}

\begin{tabular}{|c|c|c|c|c|c|}
\hline \multicolumn{6}{|c|}{$\mathbf{n}=1 \quad$ Enthalpy $=-311.843617 \mathrm{H}$} \\
\hline \multicolumn{6}{|c|}{ 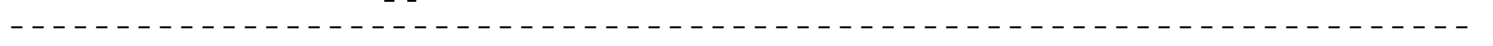 } \\
\hline Center & Atomic & Atomic & \multicolumn{3}{|c|}{ Coordinates (Angstroms) } \\
\hline Number & Number & Type & $\mathrm{X}$ & $\mathrm{Y}$ & Z \\
\hline \multicolumn{6}{|c|}{-----------------------------------------------------} \\
\hline 1 & 6 & 0 & -0.453096 & -0.283967 & 0.404199 \\
\hline 2 & 1 & 0 & -0.149883 & -0.554181 & 1.418891 \\
\hline 3 & 6 & 0 & -1.869644 & -0.548063 & 0.036376 \\
\hline 4 & 1 & 0 & -2.100654 & -1.552952 & -0.329114 \\
\hline 5 & 6 & 0 & 0.453116 & 0.283991 & -0.404344 \\
\hline 6 & 1 & 0 & 0.149896 & 0.554203 & -1.419035 \\
\hline 7 & 6 & 0 & 1.869660 & 0.548112 & -0.036527 \\
\hline 8 & 1 & 0 & 2.100664 & 1.553037 & 0.328865 \\
\hline 9 & 6 & 0 & 2.875457 & -0.321626 & -0.198668 \\
\hline 10 & 1 & 0 & 2.648970 & -1.315003 & -0.590575 \\
\hline 11 & 6 & 0 & -2.875426 & 0.321693 & 0.198509 \\
\hline 12 & 1 & 0 & -2.648910 & 1.315101 & 0.590321 \\
\hline 13 & 6 & 0 & 4.316709 & -0.046746 & 0.129171 \\
\hline 14 & 1 & 0 & 4.953489 & -0.163101 & -0.758708 \\
\hline 15 & 1 & 0 & 4.693931 & -0.752820 & 0.881753 \\
\hline 16 & 1 & 0 & 4.454902 & 0.969102 & 0.516491 \\
\hline 17 & 6 & 0 & -4.316752 & 0.046658 & -0.128873 \\
\hline 18 & 1 & 0 & -4.695684 & 0.755604 & -0.877852 \\
\hline 19 & 1 & 0 & -4.952585 & 0.158365 & 0.760311 \\
\hline 20 & 1 & 0 & -4.454278 & -0.967666 & -0.520403 \\
\hline
\end{tabular}




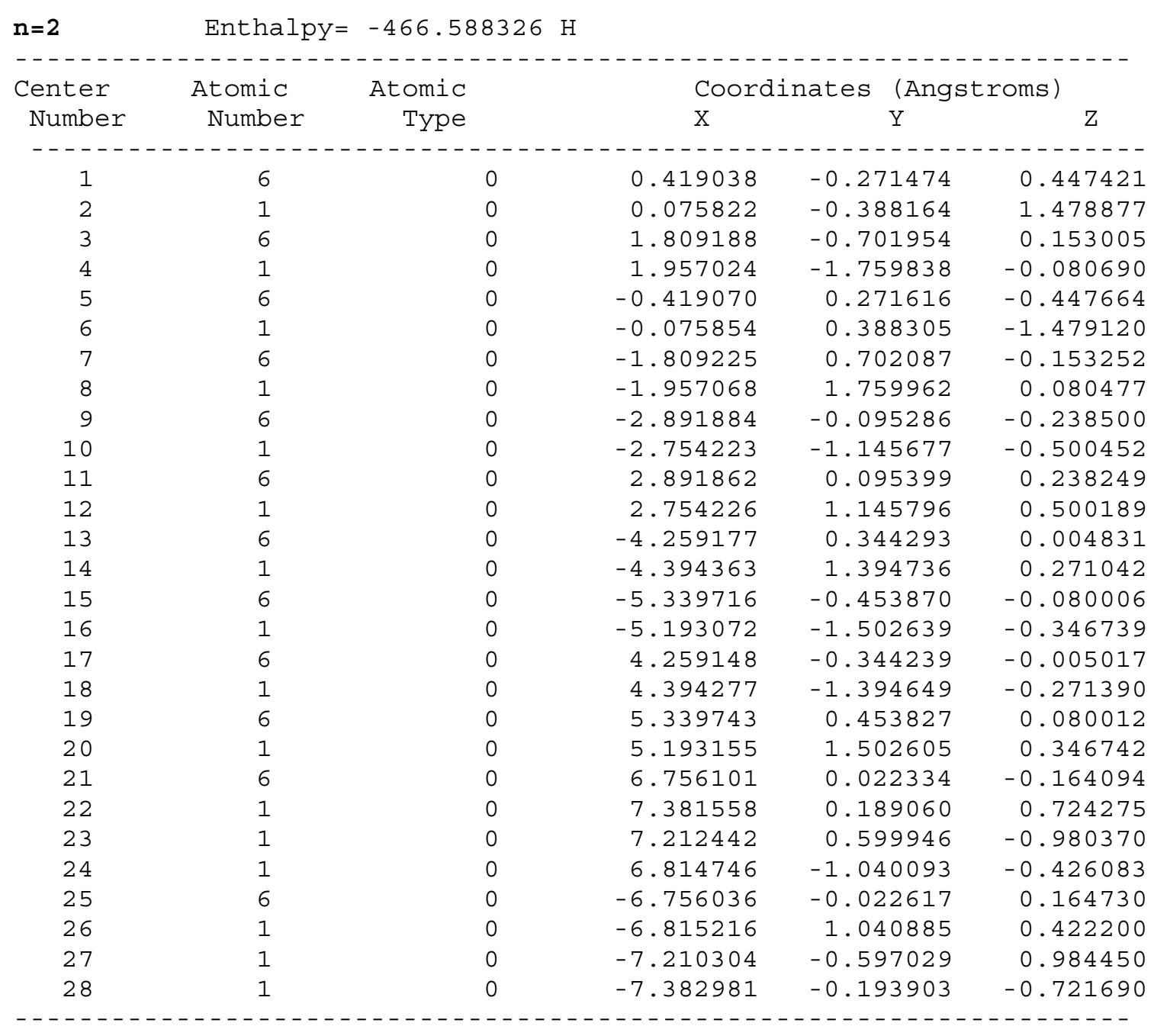




\begin{tabular}{|c|c|c|c|c|c|}
\hline$n=3$ & Enthalp & -621.3361 & & & \\
\hline------- & ------- & ------- & --------- & --------- & -------- \\
\hline Center & Atomic & Atomic & Coor & lates (Angs & coms ) \\
\hline Number & Number & Type & $\mathrm{X}$ & $\mathrm{Y}$ & $\mathrm{Z}$ \\
\hline------ & ------- & ------ & ---------- & ---------- & --------- \\
\hline 1 & 6 & 0 & 0.401570 & -0.277320 & 0.460104 \\
\hline 2 & 1 & 0 & 0.042203 & -0.349351 & 1.490285 \\
\hline 3 & 6 & 0 & 1.775346 & -0.773279 & 0.196906 \\
\hline 4 & 1 & 0 & 1.882995 & -1.843685 & 0.001955 \\
\hline 5 & 6 & 0 & -0.401536 & 0.277018 & -0.459880 \\
\hline 6 & 1 & 0 & -0.042162 & 0.349065 & -1.490057 \\
\hline 7 & 6 & 0 & -1.775297 & 0.773007 & -0.196667 \\
\hline 8 & 1 & 0 & -1.882908 & 1.843411 & -0.001681 \\
\hline 9 & 6 & 0 & -2.890716 & 0.016980 & -0.273224 \\
\hline 10 & 1 & 0 & -2.791006 & -1.045906 & -0.499387 \\
\hline 11 & 6 & 0 & 2.890732 & -0.017202 & 0.273448 \\
\hline 12 & 1 & 0 & 2.790967 & 1.045684 & 0.499586 \\
\hline 13 & 6 & 0 & -4.236182 & 0.517246 & -0.067823 \\
\hline 14 & 1 & 0 & -4.336331 & 1.579152 & 0.164324 \\
\hline 15 & 6 & 0 & 4.236227 & -0.517387 & 0.068041 \\
\hline 16 & 1 & 0 & 4.336473 & -1.579352 & -0.163794 \\
\hline 17 & 6 & 0 & 5.354134 & 0.244728 & 0.149218 \\
\hline 18 & 1 & 0 & 5.250577 & 1.306446 & 0.381298 \\
\hline 19 & 6 & 0 & -5.354171 & -0.244710 & -0.149357 \\
\hline 20 & 1 & 0 & -5.250724 & -1.306376 & -0.381722 \\
\hline 21 & 6 & 0 & -6.702146 & 0.250028 & 0.052176 \\
\hline 22 & 1 & 0 & -6.803201 & 1.312229 & 0.284712 \\
\hline 23 & 6 & 0 & 6.702150 & -0.249890 & -0.052325 \\
\hline 24 & 1 & 0 & 6.803329 & -1.312145 & -0.284561 \\
\hline 25 & 6 & 0 & 7.814134 & 0.508711 & 0.027064 \\
\hline 26 & 1 & 0 & 7.705214 & 1.570106 & 0.259399 \\
\hline 27 & 6 & 0 & -7.814229 & -0.508383 & -0.027630 \\
\hline 28 & 1 & 0 & -7.705439 & -1.569691 & -0.260424 \\
\hline 29 & 6 & 0 & -9.214775 & -0.013360 & 0.179840 \\
\hline 30 & 1 & 0 & -9.234925 & 1.059050 & 0.404211 \\
\hline 31 & 1 & 0 & -9.705699 & -0.543497 & 1.008409 \\
\hline 32 & 1 & 0 & -9.834375 & -0.186455 & -0.711533 \\
\hline 33 & 6 & 0 & 9.214756 & 0.013713 & -0.179943 \\
\hline 34 & 1 & 0 & 9.833075 & 0.183591 & 0.712969 \\
\hline 35 & 1 & 0 & 9.707197 & 0.546317 & -1.005991 \\
\hline 36 & 1 & 0 & 9.234742 & -1.057987 & -0.407694 \\
\hline
\end{tabular}




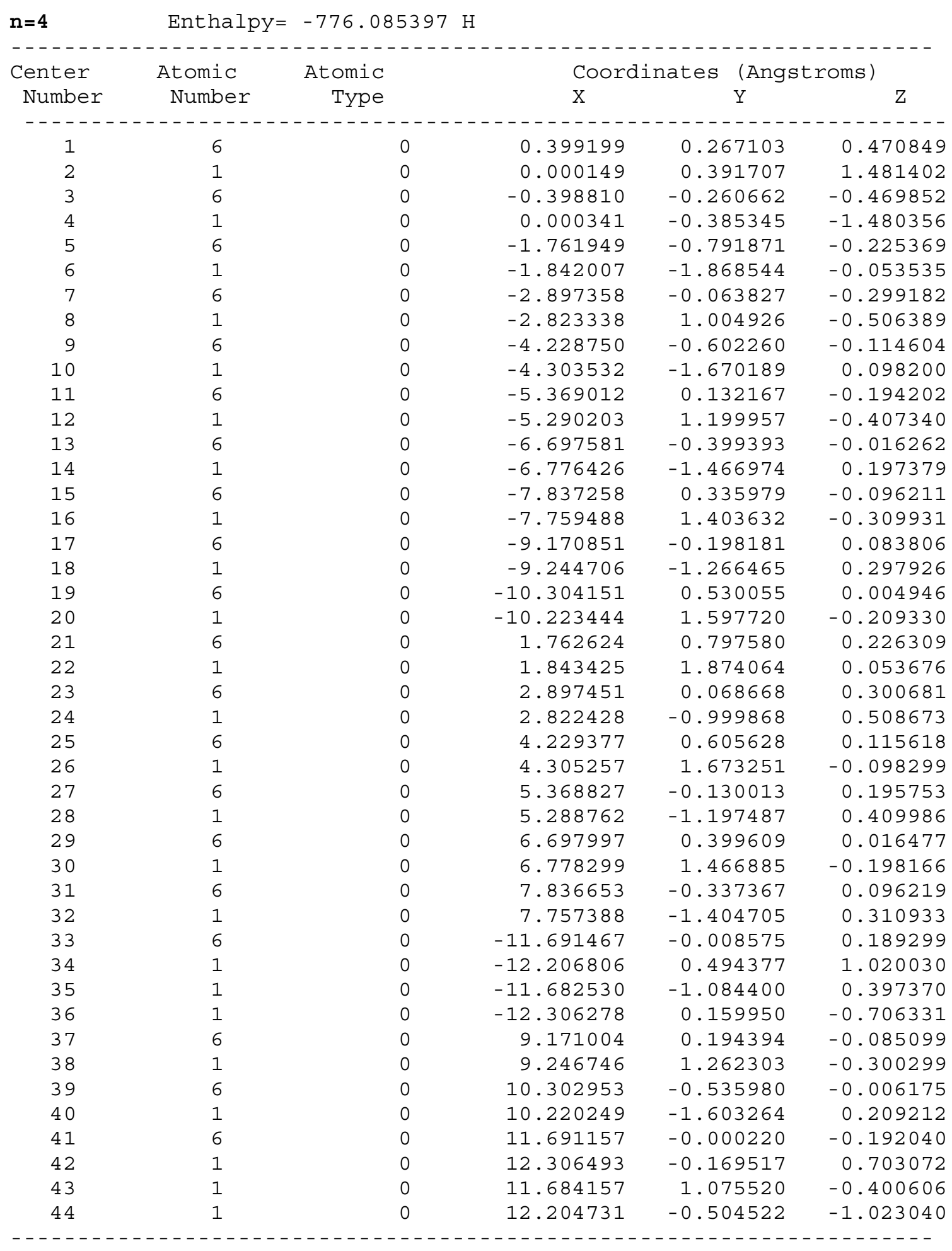




\section{Linear Perpendicular Dication}

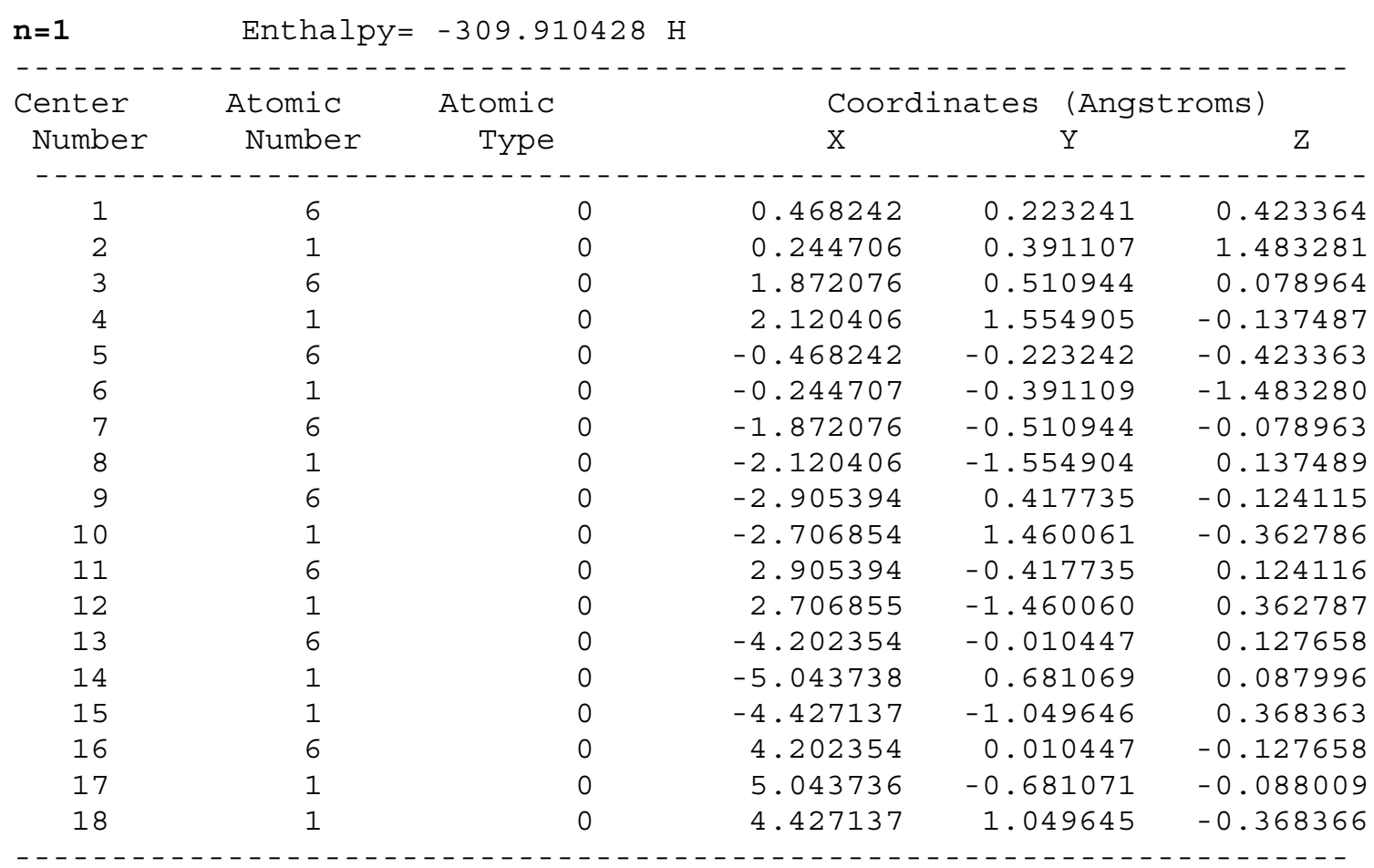




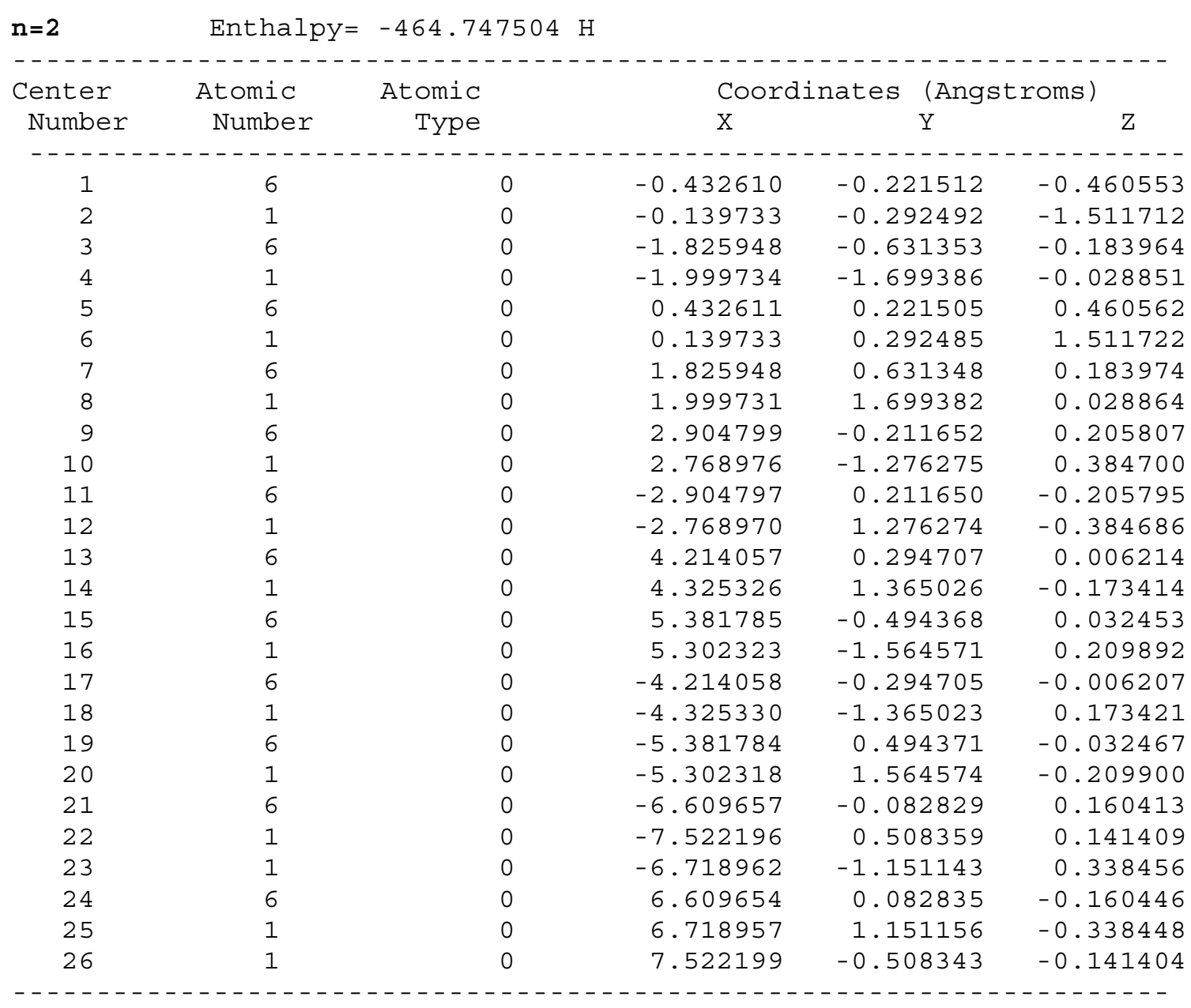




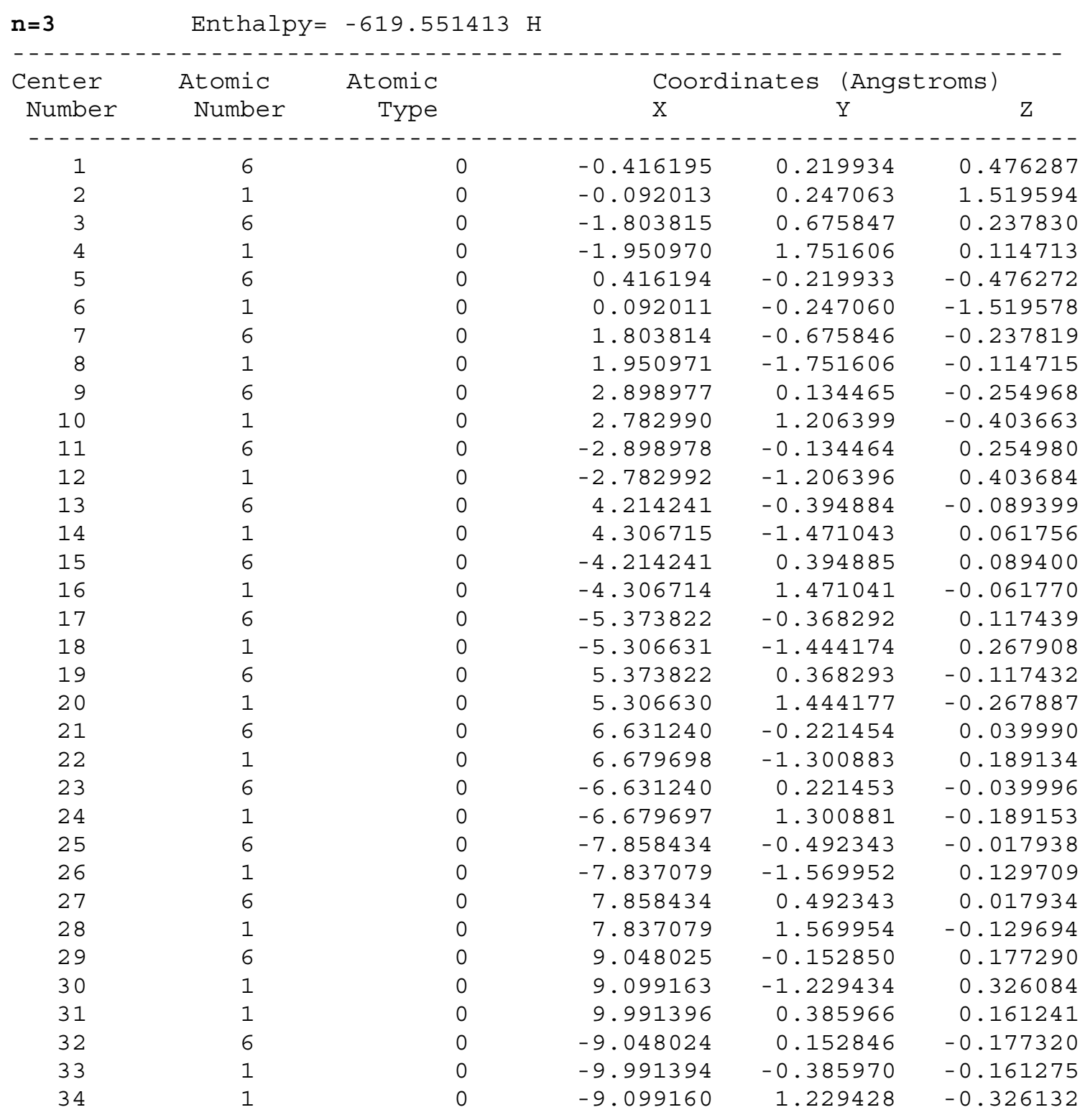




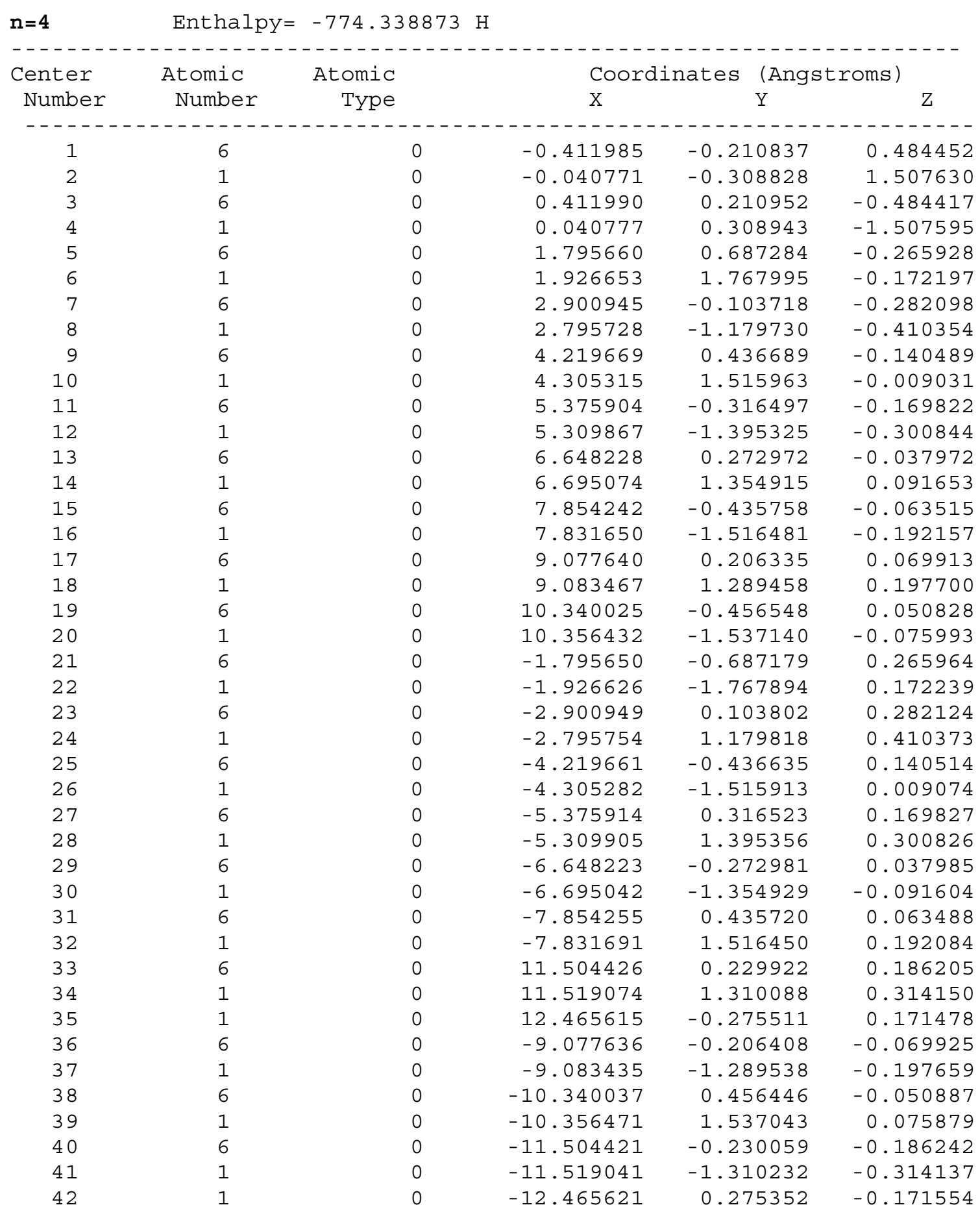




\section{Linear Reference Neutral}

\begin{tabular}{|c|c|c|c|c|c|}
\hline & Enthalpy = & \multicolumn{2}{|c|}{$-158.324178 \mathrm{H}$} & \\
\hline \multicolumn{6}{|c|}{ - - - - - - - - - - - - - - - - - - - - - - - - - - - - - - - - - - - - - - - - - - - - - - - - - - - - - - - - - - - - - - - - - - } \\
\hline Center & Atomic & Atomic & \multicolumn{3}{|c|}{ Coordinates (Angstroms) } \\
\hline Number & Number & Type & $\mathrm{X}$ & $\mathrm{Y}$ & $\mathrm{Z}$ \\
\hline \multicolumn{6}{|c|}{--------------------------------------------------------------------} \\
\hline 1 & 6 & 0 & 0.704727 & 1.836854 & 0.000000 \\
\hline 2 & 1 & 0 & 0.190766 & 2.233371 & 0.885348 \\
\hline 3 & 1 & 0 & 0.190766 & 2.233371 & -0.885348 \\
\hline 4 & 1 & 0 & 1.725310 & 2.238685 & 0.000000 \\
\hline 5 & 6 & 0 & 0.704727 & 0.303934 & 0.000000 \\
\hline 6 & 1 & 0 & 1.255571 & -0.062683 & 0.878843 \\
\hline 7 & 1 & 0 & 1.255571 & -0.062683 & -0.878843 \\
\hline 8 & 6 & 0 & -0.704727 & -0.303934 & 0.000000 \\
\hline 9 & 1 & 0 & -1.255571 & 0.062683 & 0.878843 \\
\hline 10 & 1 & 0 & -1.255571 & 0.062683 & -0.878843 \\
\hline 11 & 6 & 0 & -0.704727 & -1.836854 & 0.000000 \\
\hline 12 & 1 & 0 & -0.190766 & -2.233371 & 0.885348 \\
\hline 13 & 1 & 0 & -0.190766 & -2.233371 & -0.885348 \\
\hline 14 & 1 & 0 & -1.725310 & -2.238685 & 0.000000 \\
\hline
\end{tabular}

\begin{tabular}{|c|c|c|c|c|c|}
\hline $\mathrm{n}=1$ & Enthalp & 313.048 & & & \\
\hline-------- & ------ & ------ & --------- & --------- & --------- \\
\hline Center & Atomic & Atomic & $\mathrm{COO}$ & nates (An & Eroms ) \\
\hline Number & Number & Type & $\mathrm{X}$ & $\mathrm{Y}$ & Z \\
\hline------ & ---- & ------- & ------- & -------- & --------- \\
\hline 1 & 6 & 0 & 0.585547 & -0.497363 & -0.000001 \\
\hline 2 & 6 & 0 & -0.585547 & 0.497364 & -0.000003 \\
\hline 3 & 1 & 0 & -0.495563 & 1.153833 & 0.878163 \\
\hline 4 & 1 & 0 & -0.495562 & 1.153842 & -0.878161 \\
\hline 5 & 6 & 0 & 1.940427 & 0.182082 & 0.000001 \\
\hline 6 & 1 & 0 & 1.933452 & 1.275048 & 0.000010 \\
\hline 7 & 6 & 0 & 3.122441 & -0.445320 & 0.000001 \\
\hline 8 & 1 & 0 & 3.139121 & -1.538444 & -0.000004 \\
\hline 9 & 6 & 0 & -1.940427 & -0.182081 & -0.000006 \\
\hline 10 & 1 & 0 & -1.933453 & -1.275047 & -0.000005 \\
\hline 11 & 6 & 0 & -3.122442 & 0.445320 & -0.000004 \\
\hline 12 & 1 & 0 & -3.139124 & 1.538444 & -0.000007 \\
\hline 13 & 6 & 0 & -4.464560 & -0.232769 & 0.000006 \\
\hline 14 & 1 & 0 & -5.056046 & 0.052795 & -0.881133 \\
\hline 15 & 1 & 0 & -5.055947 & 0.052615 & 0.881270 \\
\hline 16 & 1 & 0 & -4.362061 & -1.324107 & -0.000114 \\
\hline 17 & 6 & 0 & 4.464561 & 0.232767 & 0.000001 \\
\hline 18 & 1 & 0 & 5.055974 & -0.052661 & -0.881231 \\
\hline 19 & 1 & 0 & 5.056019 & -0.052755 & 0.881173 \\
\hline 20 & 1 & 0 & 4.362064 & 1.324105 & 0.000063 \\
\hline 21 & 1 & 0 & 0.495561 & -1.153833 & 0.878164 \\
\hline 22 & 1 & 0 & 0.495564 & -1.153840 & -0.878161 \\
\hline
\end{tabular}




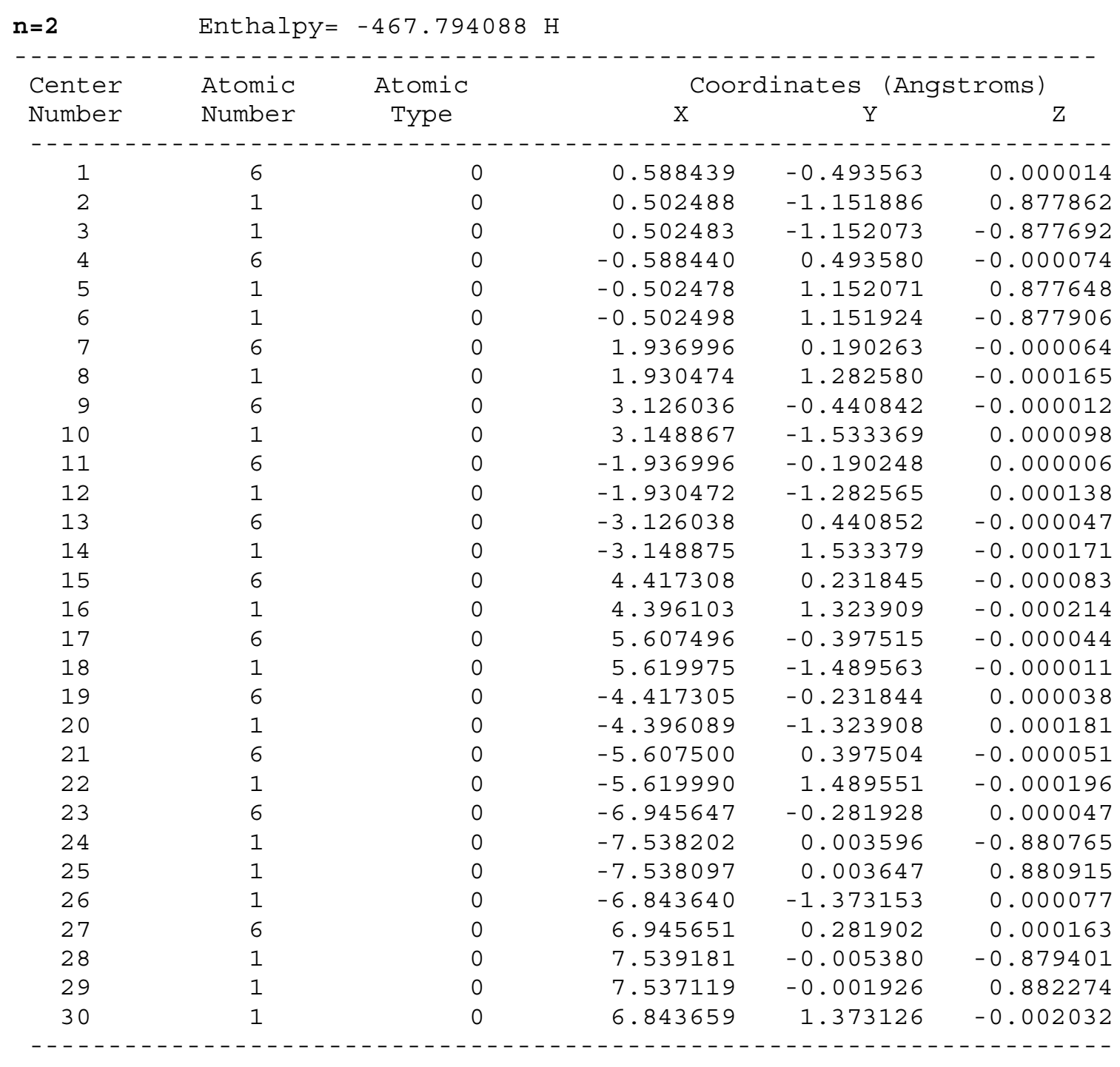




\begin{tabular}{|c|c|c|c|c|c|}
\hline $\mathrm{n}=3$ & \multicolumn{5}{|c|}{ Enthalpy $=-622.542094 \mathrm{H}$} \\
\hline------- & & & ---------- & --------- & -------- \\
\hline \multirow{2}{*}{$\begin{array}{l}\text { Center } \\
\text { Number }\end{array}$} & \multirow{2}{*}{$\begin{array}{l}\text { Atomic } \\
\text { Number }\end{array}$} & \multirow{2}{*}{$\begin{array}{l}\text { Atomic } \\
\text { Type }\end{array}$} & \multicolumn{3}{|c|}{ Coordinates (Angstroms) } \\
\hline & & & $\mathrm{X}$ & $\mathrm{Y}$ & Z \\
\hline \multicolumn{6}{|c|}{-----------------------------------------------------} \\
\hline 1 & 6 & 0 & 0.589818 & 0.491912 & -0.000059 \\
\hline 2 & 1 & 0 & 0.505691 & 1.150937 & -0.877748 \\
\hline 3 & 1 & 0 & 0.505717 & 1.150909 & 0.877653 \\
\hline 4 & 6 & 0 & -0.589816 & -0.491873 & -0.000040 \\
\hline 5 & 1 & 0 & -0.505716 & -1.150903 & -0.877728 \\
\hline 6 & 1 & 0 & -0.505683 & -1.150866 & 0.877674 \\
\hline 7 & 6 & 0 & 1.935810 & -0.194681 & -0.000082 \\
\hline 8 & 1 & 0 & 1.928562 & -1.286840 & -0.000062 \\
\hline 9 & 6 & 0 & 3.127323 & 0.436944 & -0.000104 \\
\hline 10 & 1 & 0 & 3.148134 & 1.529453 & -0.000125 \\
\hline 11 & 6 & 0 & -1.935810 & 0.194715 & -0.000018 \\
\hline 12 & 1 & 0 & -1.928567 & 1.286874 & 0.000025 \\
\hline 13 & 6 & 0 & -3.127320 & -0.436916 & 0.000003 \\
\hline 14 & 1 & 0 & -3.148124 & -1.529426 & -0.000023 \\
\hline 15 & 6 & 0 & 4.413057 & -0.231309 & -0.000099 \\
\hline 16 & 1 & 0 & 4.398345 & -1.322948 & -0.000124 \\
\hline 17 & 6 & 0 & 5.609538 & 0.406485 & -0.000070 \\
\hline 18 & 1 & 0 & 5.623346 & 1.498113 & -0.000066 \\
\hline 19 & 6 & 0 & -4.413060 & 0.231325 & 0.000042 \\
\hline 20 & 1 & 0 & -4.398360 & 1.322964 & 0.000088 \\
\hline 21 & 6 & 0 & -5.609533 & -0.406483 & 0.000024 \\
\hline 22 & 1 & 0 & -5.623328 & -1.498111 & -0.000019 \\
\hline 23 & 6 & 0 & -6.896141 & 0.261757 & 0.000054 \\
\hline 24 & 1 & 0 & -6.878701 & 1.353766 & 0.000100 \\
\hline 25 & 6 & 0 & -8.087550 & -0.370029 & 0.000030 \\
\hline 26 & 1 & 0 & -8.098551 & -1.461939 & -0.000011 \\
\hline 27 & 6 & 0 & 6.896137 & -0.261773 & -0.000038 \\
\hline 28 & 1 & 0 & 6.878680 & -1.353781 & -0.000102 \\
\hline 29 & 6 & 0 & 8.087555 & 0.369994 & 0.000063 \\
\hline 30 & 1 & 0 & 8.098573 & 1.461904 & 0.000100 \\
\hline 31 & 6 & 0 & 9.425108 & -0.309003 & 0.000183 \\
\hline 32 & 1 & 0 & 10.017370 & -0.023546 & 0.881311 \\
\hline 33 & 1 & 0 & 10.018065 & -0.022526 & -0.880136 \\
\hline 34 & 1 & 0 & 9.323454 & -1.400185 & -0.000468 \\
\hline 35 & 6 & 0 & -9.425114 & 0.308948 & 0.000038 \\
\hline 36 & 1 & 0 & -10.017809 & 0.022788 & 0.880640 \\
\hline 37 & 1 & 0 & -10.017628 & 0.023157 & -0.880808 \\
\hline 38 & 1 & 0 & -9.323475 & 1.400132 & 0.000271 \\
\hline
\end{tabular}




\begin{tabular}{|c|c|c|c|c|c|}
\hline $\mathrm{n}=4$ & Enthalp & 777.290 & & & \\
\hline-------- & $-2-3-2-1$ & - _ - - - - - & ---------- & -------- & --------- \\
\hline Center & Atomic & Atomic & Coor & nates (Ang & Eroms ) \\
\hline Number & Number & Type & $\mathrm{X}$ & $\mathrm{Y}$ & Z \\
\hline------- & ------- & ------ & ----------- & --------- & ---------- \\
\hline 1 & 6 & 0 & -0.589673 & -0.485200 & -0.000030 \\
\hline 2 & 6 & 0 & 0.590244 & 0.498071 & -0.000036 \\
\hline 3 & 1 & 0 & 0.507009 & 1.157255 & 0.877663 \\
\hline 4 & 1 & 0 & 0.507010 & 1.157246 & -0.877741 \\
\hline 5 & 6 & 0 & 1.935038 & -0.190180 & -0.000035 \\
\hline 6 & 1 & 0 & 1.926700 & -1.282281 & -0.000028 \\
\hline 7 & 6 & 0 & 3.128248 & 0.440362 & -0.000039 \\
\hline 8 & 1 & 0 & 3.150142 & 1.532838 & -0.000045 \\
\hline 9 & 6 & 0 & 4.410694 & -0.229314 & -0.000033 \\
\hline 10 & 1 & 0 & 4.395314 & -1.320871 & -0.000043 \\
\hline 11 & 6 & 0 & 5.610884 & 0.408242 & -0.000016 \\
\hline 12 & 1 & 0 & 5.623388 & 1.499897 & -0.000007 \\
\hline 13 & 6 & 0 & 6.890315 & -0.256262 & -0.000009 \\
\hline 14 & 1 & 0 & 6.878660 & -1.347886 & -0.000018 \\
\hline 15 & 6 & 0 & 8.089273 & 0.383118 & 0.000008 \\
\hline 16 & 1 & 0 & 8.102020 & 1.474664 & 0.000017 \\
\hline 17 & 6 & 0 & 9.373869 & -0.284464 & 0.000017 \\
\hline 18 & 1 & 0 & 9.356901 & -1.376448 & 0.000000 \\
\hline 19 & 6 & 0 & 10.566016 & 0.347868 & 0.000044 \\
\hline 20 & 1 & 0 & 10.576664 & 1.439733 & 0.000060 \\
\hline 21 & 6 & 0 & -1.936204 & 0.199269 & -0.000025 \\
\hline 22 & 1 & 0 & -1.931669 & 1.291319 & -0.000023 \\
\hline 23 & 6 & 0 & -3.126933 & -0.436076 & -0.000018 \\
\hline 24 & 1 & 0 & -3.144796 & -1.528567 & -0.000013 \\
\hline 25 & 6 & 0 & -4.411462 & 0.229421 & -0.000011 \\
\hline 26 & 1 & 0 & -4.398788 & 1.321016 & -0.000006 \\
\hline 27 & 6 & 0 & -5.610526 & -0.410202 & -0.000003 \\
\hline 28 & 1 & 0 & -5.621749 & -1.501857 & 0.000001 \\
\hline 29 & 6 & 0 & -6.890544 & 0.253244 & 0.000007 \\
\hline 30 & 1 & 0 & -6.879640 & 1.344871 & 0.000008 \\
\hline 31 & 6 & 0 & -8.089053 & -0.386951 & 0.000015 \\
\hline 32 & 1 & 0 & -8.101014 & -1.478500 & 0.000018 \\
\hline 33 & 6 & 0 & -9.374152 & 0.279629 & 0.000022 \\
\hline 34 & 1 & 0 & -9.358036 & 1.371629 & 0.000022 \\
\hline 35 & 6 & 0 & -10.565805 & -0.353607 & 0.000028 \\
\hline 36 & 1 & 0 & -10.575621 & -1.445477 & 0.000029 \\
\hline 37 & 1 & 0 & -0.505384 & -1.144450 & -0.877615 \\
\hline 38 & 1 & 0 & -0.505375 & -1.144450 & 0.877553 \\
\hline 39 & 6 & 0 & -11.903566 & 0.324209 & 0.000036 \\
\hline 40 & 1 & 0 & -12.495925 & 0.037419 & 0.880697 \\
\hline 41 & 1 & 0 & -12.495929 & 0.037434 & -0.880627 \\
\hline 42 & 1 & 0 & -11.803048 & 1.415457 & 0.000045 \\
\hline 43 & 6 & 0 & 11.903264 & -0.330951 & 0.000056 \\
\hline 44 & 1 & 0 & 11.801919 & -1.422125 & 0.000008 \\
\hline 45 & 1 & 0 & 12.495816 & -0.044647 & 0.880745 \\
\hline 46 & 1 & 0 & 12.495866 & -0.044575 & -0.880575 \\
\hline
\end{tabular}




\section{Linear Reference Dication}

\begin{tabular}{|c|c|c|c|c|c|}
\hline $\mathrm{n}=0$ & Enthalpy $=$ & \multicolumn{2}{|c|}{$-156.249174 \mathrm{H}$} & \\
\hline \multicolumn{6}{|c|}{ 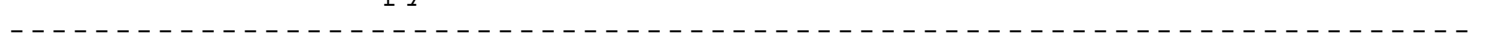 } \\
\hline Center & Atomic & Atomic & \multicolumn{3}{|c|}{ Coordinates (Angstroms) } \\
\hline Number & Number & Type & $\mathrm{X}$ & $\mathrm{Y}$ & $\mathrm{Z}$ \\
\hline \multicolumn{6}{|c|}{-----------------------------------------------------} \\
\hline 1 & 6 & 0 & 0.631811 & 0.440607 & 0.000262 \\
\hline 2 & 1 & 0 & 0.667032 & 1.179678 & -0.842059 \\
\hline 3 & 1 & 0 & 0.666022 & 1.181955 & 0.840285 \\
\hline 4 & 6 & 0 & -0.631811 & -0.440606 & 0.000436 \\
\hline 5 & 1 & 0 & -0.665799 & -1.182520 & -0.839143 \\
\hline 6 & 1 & 0 & -0.667253 & -1.179109 & 0.843200 \\
\hline 7 & 6 & 0 & -1.930540 & 0.168009 & -0.000637 \\
\hline 8 & 1 & 0 & -2.109894 & 1.249923 & -0.000152 \\
\hline 9 & 1 & 0 & -2.817965 & -0.475142 & -0.000472 \\
\hline 10 & 6 & 0 & 1.930539 & -0.168009 & -0.000432 \\
\hline 11 & 1 & 0 & 2.109892 & -1.249923 & -0.001431 \\
\hline 12 & 1 & 0 & 2.817966 & 0.475135 & 0.001992 \\
\hline
\end{tabular}

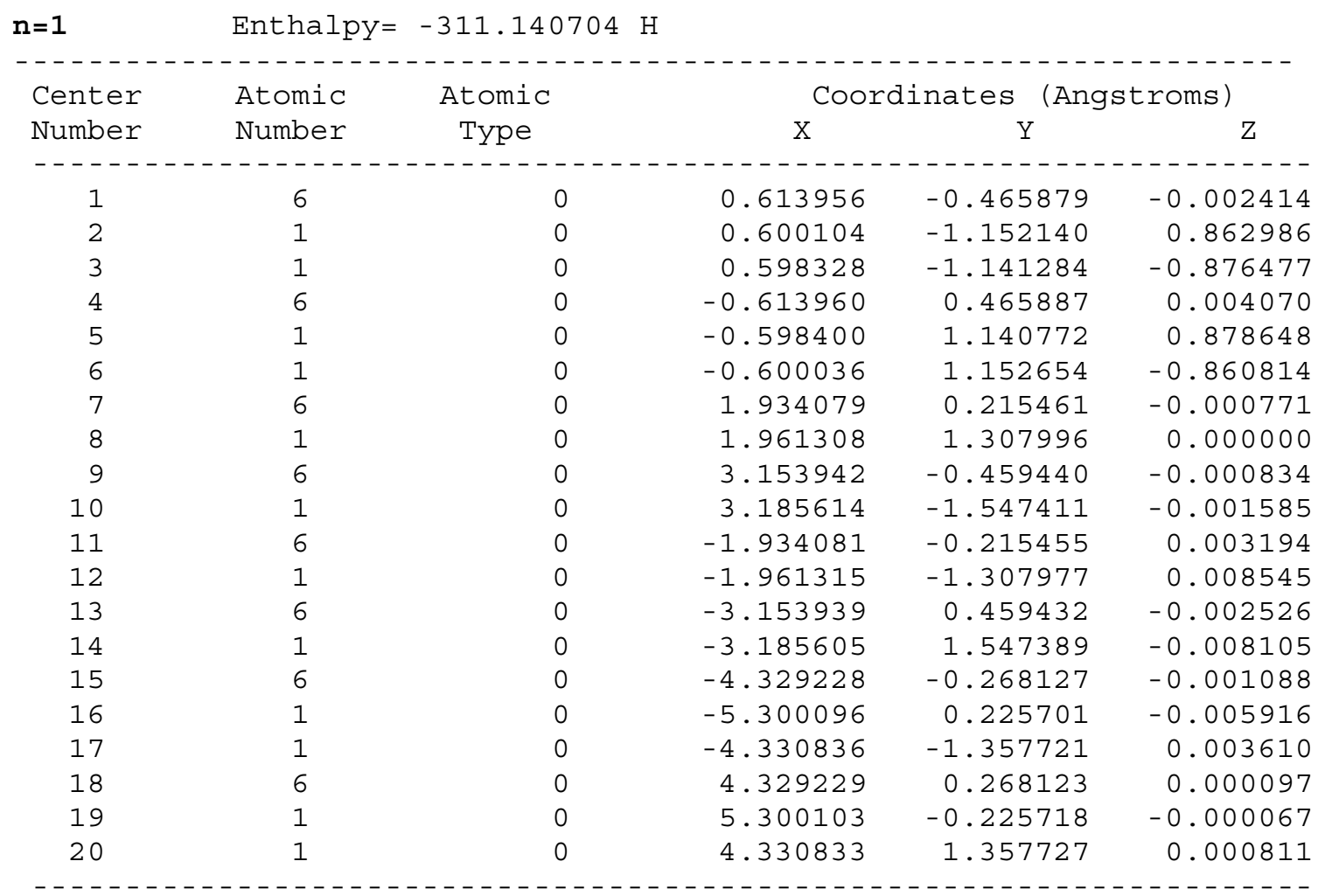




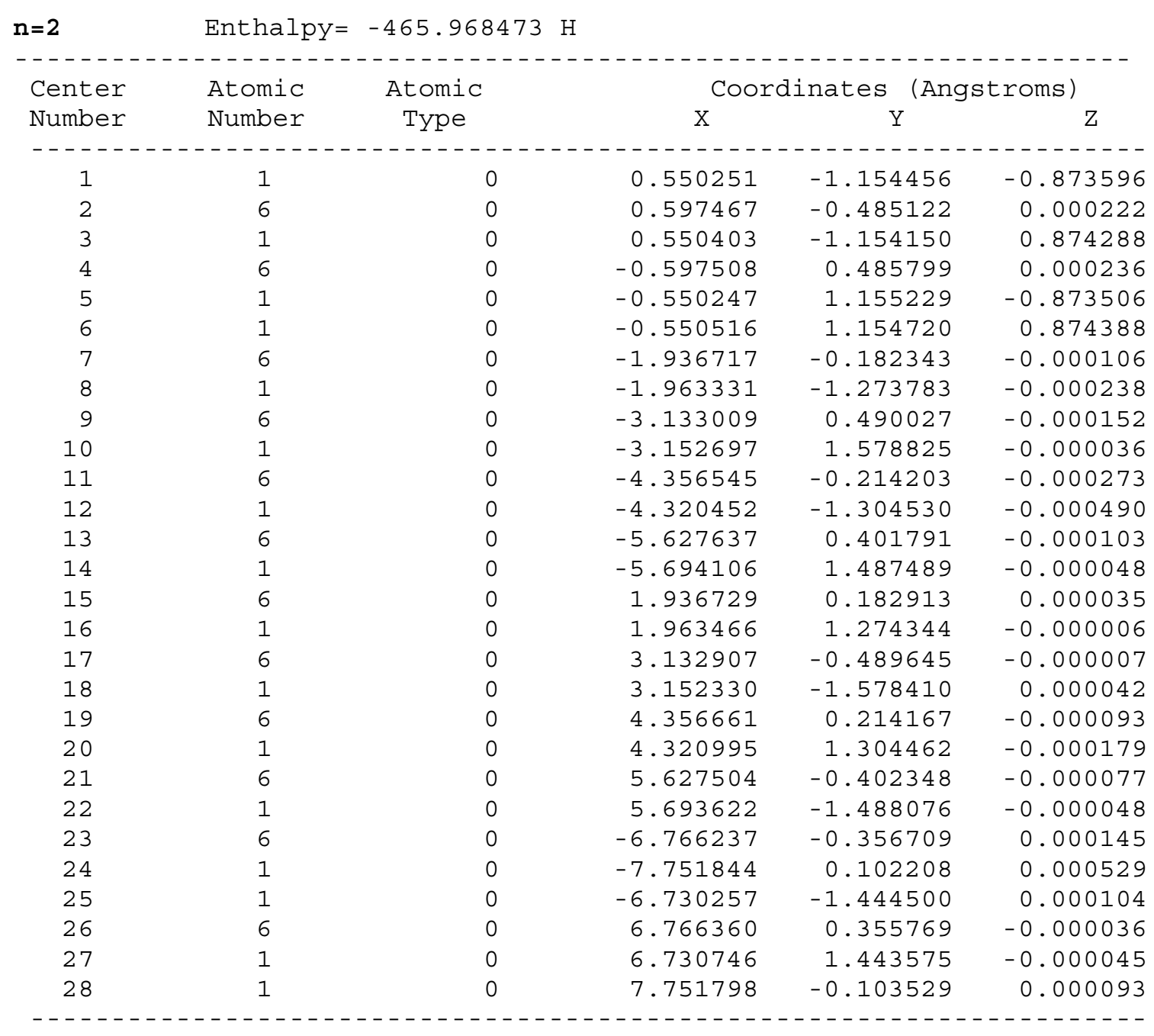




\begin{tabular}{|c|c|c|c|c|c|}
\hline & Enthalpy= & \multicolumn{2}{|c|}{$-620.769042 \mathrm{H}$} & \\
\hline------1 & & & --------- & --------1 & --------- \\
\hline \multirow{2}{*}{$\begin{array}{l}\text { Center } \\
\text { Number }\end{array}$} & \multirow{2}{*}{$\begin{array}{l}\text { Atomic } \\
\text { Number }\end{array}$} & \multirow{2}{*}{$\begin{array}{l}\text { Atomic } \\
\text { Type }\end{array}$} & \multicolumn{3}{|c|}{ Coordinates (Angstroms) } \\
\hline & & & $\mathrm{X}$ & $\mathrm{Y}$ & $\mathrm{Z}$ \\
\hline------1 & 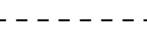 & $-\ldots-\ldots$ & --------- & --------1 & --------- \\
\hline 1 & 6 & 0 & 0.514468 & 0.343067 & 0.473587 \\
\hline 2 & 1 & 0 & 0.556332 & 1.420998 & 0.281257 \\
\hline 3 & 1 & 0 & 0.170590 & 0.212829 & 1.512082 \\
\hline 4 & 6 & 0 & -0.514414 & -0.343422 & -0.472777 \\
\hline 5 & 1 & 0 & -0.170501 & -0.213258 & -1.511270 \\
\hline 6 & 1 & 0 & -0.556320 & -1.421343 & -0.280405 \\
\hline 7 & 6 & 0 & 1.878016 & -0.260180 & 0.375103 \\
\hline 8 & 1 & 0 & 1.939659 & -1.345156 & 0.479391 \\
\hline 9 & 6 & 0 & 3.034008 & 0.441893 & 0.199216 \\
\hline 10 & 1 & 0 & 3.002994 & 1.525284 & 0.093603 \\
\hline 11 & 6 & 0 & -1.877954 & 0.259863 & -0.374377 \\
\hline 12 & 1 & 0 & -1.939558 & 1.344834 & -0.478686 \\
\hline 13 & 6 & 0 & -3.033969 & -0.442207 & -0.198618 \\
\hline 14 & 1 & 0 & -3.002995 & -1.525597 & -0.093000 \\
\hline 15 & 6 & 0 & 4.302495 & -0.204363 & 0.168273 \\
\hline 16 & 1 & 0 & 4.317263 & -1.289918 & 0.272733 \\
\hline 17 & 6 & 0 & 5.516059 & 0.458031 & 0.020659 \\
\hline 18 & 1 & 0 & 5.526569 & 1.541369 & -0.083826 \\
\hline 19 & 6 & 0 & -4.302453 & 0.204082 & -0.168034 \\
\hline 20 & 1 & 0 & -4.317108 & 1.289644 & -0.272446 \\
\hline 21 & 6 & 0 & -5.516143 & -0.458202 & -0.020865 \\
\hline 22 & 1 & 0 & -5.526846 & -1.541550 & 0.083541 \\
\hline 23 & 6 & 0 & -6.725121 & 0.239251 & -0.009935 \\
\hline 24 & 1 & 0 & -6.696506 & 1.324366 & -0.116832 \\
\hline 25 & 6 & 0 & -8.000615 & -0.371340 & 0.130154 \\
\hline 26 & 1 & 0 & -8.055026 & -1.452467 & 0.238566 \\
\hline 27 & 6 & 0 & 6.725159 & -0.239162 & 0.009490 \\
\hline 28 & 1 & 0 & 6.696848 & -1.324249 & 0.116447 \\
\hline 29 & 6 & 0 & 8.000441 & 0.371829 & -0.130889 \\
\hline 30 & 1 & 0 & 8.054589 & 1.452973 & -0.239366 \\
\hline 31 & 6 & 0 & 9.140715 & -0.372634 & -0.129514 \\
\hline 32 & 1 & 0 & 10.118629 & 0.088630 & -0.235671 \\
\hline 33 & 1 & 0 & 9.116862 & -1.455018 & -0.022010 \\
\hline 34 & 6 & 0 & -9.140712 & 0.373400 & 0.128575 \\
\hline 35 & 1 & 0 & -10.118773 & -0.087585 & 0.234484 \\
\hline 36 & 1 & 0 & -9.116591 & 1.455778 & 0.021113 \\
\hline
\end{tabular}




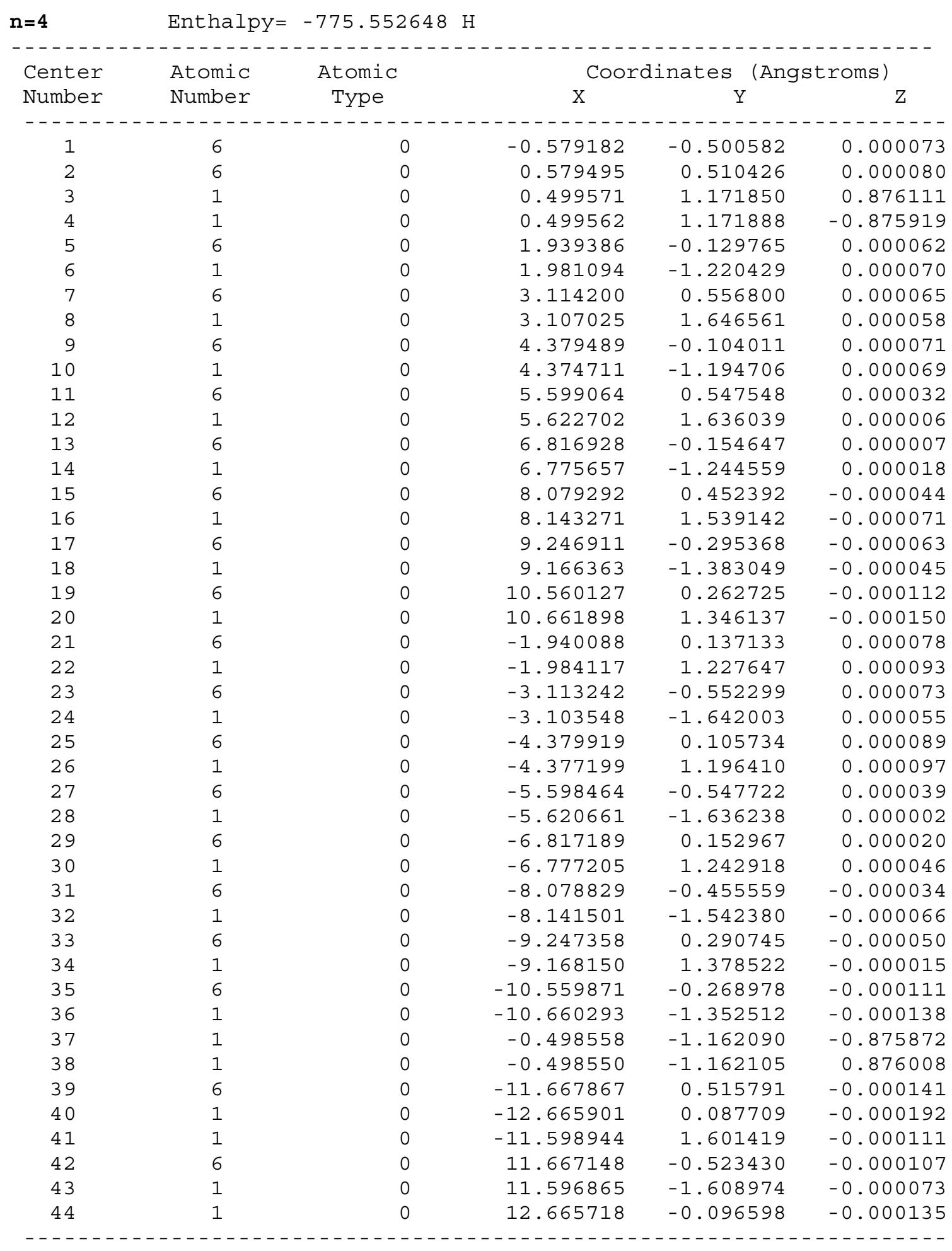


Structures involved in the ODP Methodology

\begin{tabular}{|c|c|c|c|}
\hline \multicolumn{4}{|c|}{ 6-31G(d) basis set } \\
\hline Optimal & I geometry of & delocalized & $\mathrm{TMM}^{2+}(\mathrm{D} 3 \mathrm{~h})$ \\
\hline $\mathrm{C}$ & 0.000000 & 0.000000 & 0.000000 \\
\hline $\mathrm{C}$ & 0.000000 & 1.414591 & 0.000000 \\
\hline $\mathrm{C}$ & 1.225071 & -0.707295 & 0.000000 \\
\hline $\mathrm{C}$ & -1.225071 & -0.707295 & 0.000000 \\
\hline $\mathrm{H}$ & 0.920337 & 1.980508 & 0.000000 \\
\hline $\mathrm{H}$ & -0.920337 & 1.980508 & 0.000000 \\
\hline $\mathrm{H}$ & 2.175339 & -0.193219 & 0.000000 \\
\hline $\mathrm{H}$ & 1.255002 & -1.787289 & 0.000000 \\
\hline $\mathrm{H}$ & -2.175339 & -0.193219 & 0.000000 \\
\hline $\mathrm{H}$ & -1.255002 & -1.787289 & 0.000000 \\
\hline Energy & of delocalize & ed structure: & -154.1387496 a.u. \\
\hline Energy & of localized & structure 1 : & -154.0490694 a.u. \\
\hline Energy & of localized & structure 10 & -153.9786325 a.u. \\
\hline Energy & of localized & structure 11 & -153.8546311 a.u. \\
\hline
\end{tabular}

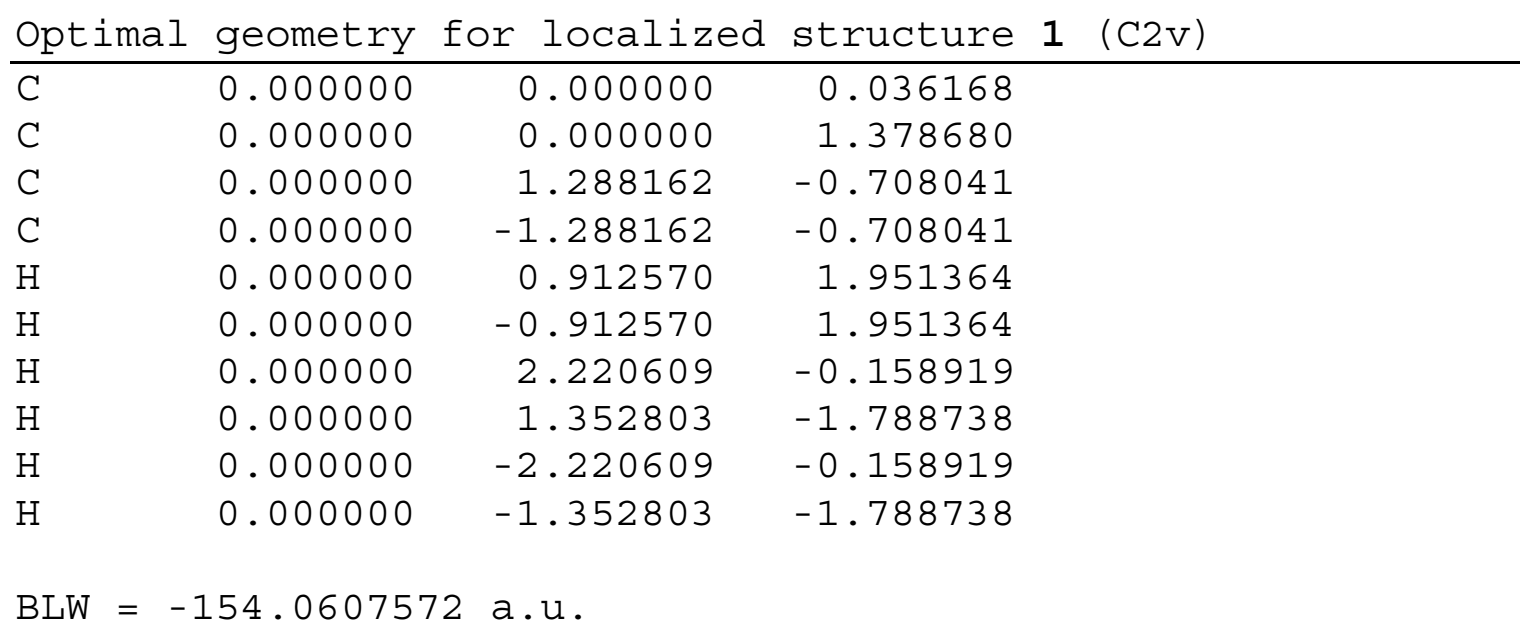

\begin{tabular}{lrrr} 
Optimal & geometry & for localized & structure 10 (D3h) \\
\hline C & 0.000000 & 0.000000 & 0.000000 \\
C & 0.000000 & 1.442276 & 0.000000 \\
C & 1.249048 & -0.721138 & 0.000000 \\
C & -1.249048 & -0.721138 & 0.000000 \\
H & 0.917226 & 2.013465 & 0.000000 \\
H & -0.917226 & 2.013465 & 0.000000 \\
H & 2.202325 & -0.212391 & 0.000000 \\
H & 1.285099 & -1.801074 & 0.000000
\end{tabular}




$\begin{array}{llll}\mathrm{H} & -2.202325 & -0.212391 & 0.000000 \\ \mathrm{H} & -1.285099 & -1.801074 & 0.000000 \\ & & \end{array}$

\begin{tabular}{lccc} 
Optimal geometry for localized & structure 11 (D3h) & \\
\hline C & 0.000000 & 0.000000 & 0.000000 \\
C & 0.000000 & 1.442983 & 0.000000 \\
C & 1.249660 & -0.721491 & 0.000000 \\
C & -1.249660 & -0.721491 & 0.000000 \\
H & 0.933838 & 1.982953 & 0.000000 \\
H & -0.933838 & 1.982953 & 0.000000 \\
H & 2.184207 & -0.182749 & 0.000000 \\
H & 1.250369 & -1.800204 & 0.000000 \\
H & -2.184207 & -0.182749 & 0.000000 \\
H & -1.250369 & -1.800204 & 0.000000
\end{tabular}

$B L W=-153.8572313 \mathrm{a} \cdot \mathrm{u}$.

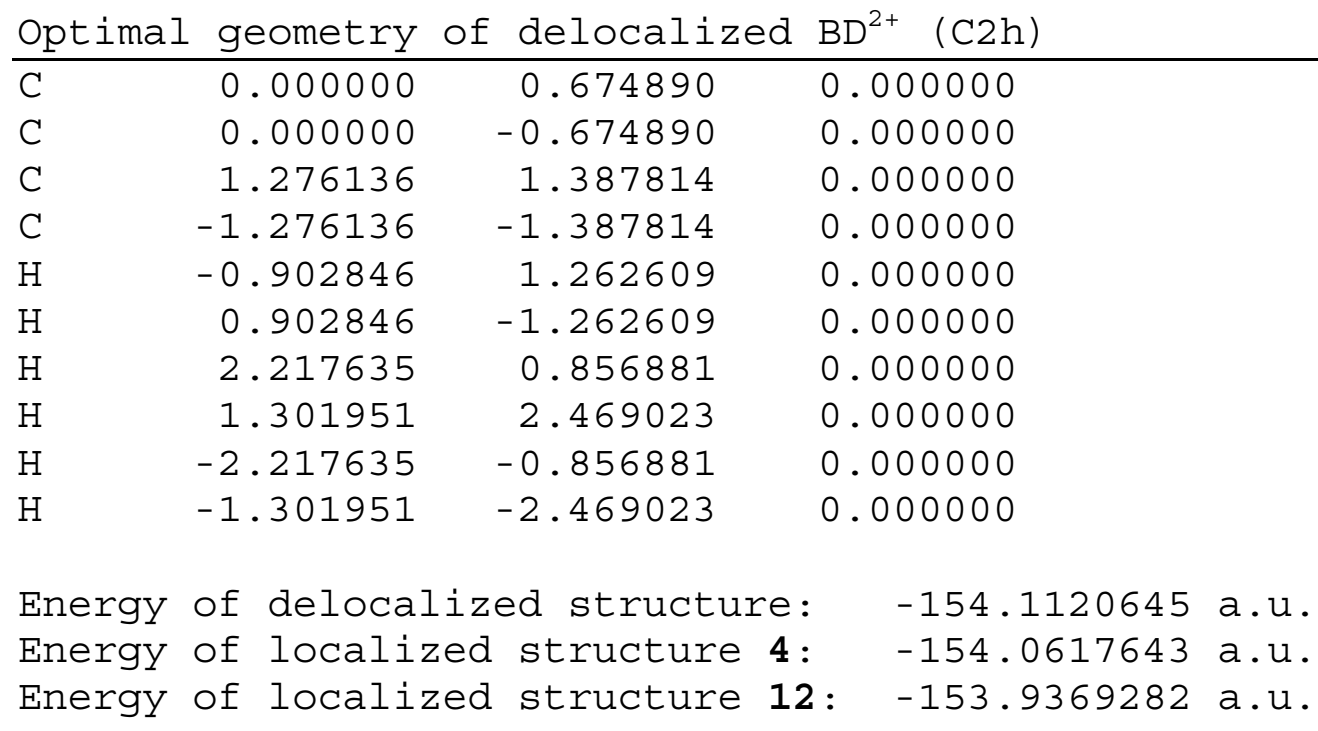

\begin{tabular}{lrrrr} 
Optimal & geometry & for localized & structure 4 & $(\mathrm{C} 2 \mathrm{~h})$ \\
\hline C & 0.000000 & 0.659649 & 0.000000 & \\
C & 0.000000 & -0.659649 & 0.000000 & \\
C & 1.306517 & 1.423215 & 0.000000 & \\
C & -1.306517 & -1.423215 & 0.000000
\end{tabular}




$\begin{array}{rrrr}\mathrm{H} & -0.889669 & 1.264865 & 0.000000 \\ \mathrm{H} & 0.889669 & -1.264865 & 0.000000 \\ \mathrm{H} & 2.260961 & 0.913234 & 0.000000 \\ \mathrm{H} & 1.306141 & 2.505651 & 0.000000 \\ \mathrm{H} & -2.260961 & -0.913234 & 0.000000 \\ \mathrm{H} & -1.306141 & -2.505651 & 0.000000\end{array}$

$B L W=-154.0663414 \mathrm{a} \cdot \mathrm{u}$.

\begin{tabular}{lrrr} 
Optimal geometry & for localized & structure 12 & (CS) \\
\hline C & 0.000000 & 0.716768 & 0.000000 \\
C & -0.133480 & -0.767965 & 0.000000 \\
C & 1.443617 & 1.374562 & 0.000000 \\
C & -1.333987 & -1.334171 & 0.000000 \\
H & -0.841152 & 1.406077 & 0.000000 \\
H & 0.784983 & -1.325189 & 0.000000 \\
H & 2.315807 & 0.724825 & 0.000000 \\
H & 1.550403 & 2.457204 & 0.000000 \\
H & -2.264228 & -0.790608 & 0.000000 \\
H & -1.402713 & -2.407471 & 0.000000 \\
BLW $=-153.9752426$ & a.u. &
\end{tabular}

\section{$6-311+G(d, p)$ basis set}

\begin{tabular}{lrrr} 
Optimal & geometry & of delocalized & $\mathrm{TMM}^{2+}(\mathrm{D} 3 \mathrm{~h}$ \\
\hline $\mathrm{C}$ & 0.000000 & 0.000000 & 0.000000 \\
$\mathrm{C}$ & 0.000000 & 1.413380 & 0.000000 \\
$\mathrm{C}$ & 1.224023 & -0.706690 & 0.000000 \\
$\mathrm{C}$ & -1.224023 & -0.706690 & 0.000000 \\
$\mathrm{H}$ & 0.921479 & 1.981532 & 0.000000 \\
$\mathrm{H}$ & -0.921479 & 1.981532 & 0.000000 \\
$\mathrm{H}$ & 2.176796 & -0.192742 & 0.000000 \\
$\mathrm{H}$ & 1.255317 & -1.788790 & 0.000000 \\
$\mathrm{H}$ & -2.176796 & -0.192742 & 0.000000 \\
$\mathrm{H}$ & -1.255317 & -1.788790 & 0.000000
\end{tabular}

$\begin{array}{lll}\text { Energy of delocalized structure: } & -154.1746608 \mathrm{a} \cdot \mathrm{u} . \\ \text { Energy of localized structure 1: } & -154.0860019 \mathrm{a} \cdot \mathrm{u} . \\ \text { Energy of localized structure 10: } & -154.0167657 \mathrm{a} . \mathrm{u} . \\ \text { Energy of localized structure 11: } & -153.8987009 \mathrm{a} \cdot \mathrm{u} .\end{array}$ 


\begin{tabular}{|c|c|c|c|}
\hline $\bar{C}$ & 0.000000 & 0.000000 & 0.039041 \\
\hline $\mathrm{C}$ & 0.000000 & 0.000000 & 1.381276 \\
\hline $\mathrm{C}$ & 0.000000 & 1.289435 & -0.709805 \\
\hline $\mathrm{C}$ & 0.000000 & -1.289435 & -0.709805 \\
\hline $\mathrm{H}$ & 0.000000 & 0.914631 & 1.953416 \\
\hline $\mathrm{H}$ & 0.000000 & -0.914631 & 1.953416 \\
\hline $\mathrm{H}$ & 0.000000 & 2.226062 & -0.161868 \\
\hline $\mathrm{H}$ & 0.000000 & 1.350196 & -1.793668 \\
\hline $\mathrm{H}$ & 0.000000 & -2.226062 & -0.161868 \\
\hline $\mathrm{H}$ & 0.000000 & -1.350196 & -1.793668 \\
\hline
\end{tabular}

\begin{tabular}{|c|c|c|c|}
\hline $\bar{C}$ & 0.000000 & 0.000000 & 0.000000 \\
\hline $\mathrm{C}$ & 0.000000 & 1.448484 & 0.000000 \\
\hline $\mathrm{C}$ & 1.254424 & -0.724242 & 0.000000 \\
\hline $\mathrm{C}$ & -1.254424 & -0.724242 & 0.000000 \\
\hline $\mathrm{H}$ & 0.920407 & 2.020570 & 0.000000 \\
\hline $\mathrm{H}$ & -0.920407 & 2.020570 & 0.000000 \\
\hline $\mathrm{H}$ & 2.210068 & -0.213189 & 0.000000 \\
\hline $\mathrm{H}$ & 1.289661 & -1.807380 & 0.000000 \\
\hline $\mathrm{H}$ & -2.210068 & -0.213189 & 0.000000 \\
\hline $\mathrm{H}$ & -1.289661 & -1.807380 & 0.000000 \\
\hline
\end{tabular}

\begin{tabular}{lccc} 
Optimal geometry for localized & structure 11 & (D3h) \\
\hline C & 0.000000 & 0.000000 & 0.000000 \\
C & 0.000000 & 1.444547 & 0.000000 \\
C & 1.251014 & -0.722274 & 0.000000 \\
C & -1.251014 & -0.722274 & 0.000000 \\
H & 0.937113 & 1.983626 & 0.000000 \\
H & -0.937113 & 1.983626 & 0.000000 \\
H & 2.186427 & -0.180250 & 0.000000 \\
H & 1.249314 & -1.803376 & 0.000000 \\
H & -2.186427 & -0.180250 & 0.000000 \\
H & -1.249314 & -1.803376 & 0.000000
\end{tabular}

$B L W=-153.9017087 \mathrm{a} \cdot \mathrm{u}$. 


\begin{tabular}{lrrr} 
Optimal geometry & of delocalized & \multicolumn{1}{l}{$\mathrm{BD}^{2+}(\mathrm{C} 2 \mathrm{~h})$} \\
\hline $\mathrm{C}$ & 0.000000 & 0.673827 & 0.000000 \\
$\mathrm{C}$ & 0.000000 & -0.673827 & 0.000000 \\
$\mathrm{C}$ & 1.277846 & 1.386379 & 0.000000 \\
$\mathrm{C}$ & -1.277846 & -1.386379 & 0.000000 \\
$\mathrm{H}$ & -0.903334 & 1.262384 & 0.000000 \\
$\mathrm{H}$ & 0.903334 & -1.262384 & 0.000000 \\
$\mathrm{H}$ & 2.220710 & 0.852612 & 0.000000 \\
$\mathrm{H}$ & 1.305535 & 2.470189 & 0.000000 \\
$\mathrm{H}$ & -2.220710 & -0.852612 & 0.000000 \\
$\mathrm{H}$ & -1.305535 & -2.470189 & 0.000000
\end{tabular}

Energy of delocalized structure: $\quad-154.1480927 \mathrm{a} . \mathrm{u}$. Energy of localized structure 4: $\quad-154.0989053 \mathrm{a} . \mathrm{u}$. Energy of localized structure 12: $\quad-153.9771694$ a.u.

\begin{tabular}{lrrr} 
Optimal geometry & for localized & structure 4 & (C2h) \\
\hline C & 0.000000 & 0.659024 & 0.000000 \\
C & 0.000000 & -0.659024 & 0.000000 \\
C & 1.310190 & 1.421042 & 0.000000 \\
C & -1.310190 & -1.421042 & 0.000000 \\
H & -0.889552 & 1.265951 & 0.000000 \\
H & 0.889552 & -1.265951 & 0.000000 \\
H & 2.266154 & 0.907500 & 0.000000 \\
H & 1.312308 & 2.506528 & 0.000000 \\
H & -2.266154 & -0.907500 & 0.000000 \\
H & -1.312308 & -2.506528 & 0.000000 \\
BLW $=$ & -154.1034323 & a.u.
\end{tabular}

\begin{tabular}{lrrrr} 
Optimal geometry & for localized & structure 12 & (Cs) \\
\hline C & 0.000000 & 0.716152 & 0.000000 & \\
C & -0.136301 & -0.766934 & 0.000000 & \\
C & 1.450624 & 1.368876 & 0.000000 & \\
C & -1.338881 & -1.329653 & 0.000000 & \\
H & -0.840241 & 1.410927 & 0.000000 \\
H & 0.782186 & -1.325802 & 0.000000 \\
H & 2.321779 & 0.712458 & 0.000000
\end{tabular}




$\begin{array}{lrrr}\mathrm{H} & 1.563112 & 2.454328 & 0.000000 \\ \mathrm{H} & -2.266026 & -0.778454 & 0.000000 \\ \mathrm{H} & -1.413466 & -2.404101 & 0.000000 \\ \text { BLW }=-154.014992546 \mathrm{a} . \mathrm{u} . & \end{array}$

